\title{
Presenting the frame of the unit circle
}

\author{
Javier Gutiérrez García ${ }^{\mathrm{a}}$, Imanol Mozo Carollo ${ }^{\mathrm{a}, *}$, Jorge Picado $^{\mathrm{b}}$ \\ ${ }^{a}$ Department of Mathematics, University of the Basque Country UPV/EHU, Apdo. 644, \\ 48080 Bilbao, Spain \\ ${ }^{b}$ CMUC, Department of Mathematics, University of Coimbra, 3001-501 Coimbra, Portugal
}

\begin{abstract}
We present the frame $\mathfrak{L}(\mathbb{T})$ of the unit circle by generators and relations in two alternative ways. The first is the localic counterpart of the Alexandroff compactification of the real line while the other can be understood as a localic analogue of the quotient space $\mathbb{R} / \mathbb{Z}$. With an eye towards a prospective point-free description of Pontryagin duality, we then show how the usual group operations of the frame of reals can be lifted to the new frame $\mathfrak{L}(\mathbb{T})$, endowing it with a canonical localic group structure.
\end{abstract}

Keywords: Locale, frame, frame of reals, localic group, continuous frame, frame compactification, Alexandroff extension, Alexandroff compactification, cocompact element, frame of the unit circle.

2010 MSC: 06D22, 18D35, 22A99, 54H11

\section{Introduction}

One of the main differences between localic topology and classical topology is that the category of localic spaces (i.e., the category of locales and localic maps) is a category whose dual category (i.e., the category of frames and frame homomorphisms) is an algebraic category [13]. This means, in particular, that one has free frames and quotient frames, and therefore that one can present frames and locales by generators and relations, exactly in the same way as with

\footnotetext{
${ }^{2}$ We acknowledge financial assistance from the Ministry of Economy and Competitiveness of Spain (through grant MTM2012-37894-C02-02), the University of the Basque Country UPV/EHU (through grant GIU12/39) and the Centre for Mathematics of the University of Coimbra (funded by the European Regional Development Fund through the program COMPETE and by the Portuguese Government through the Fundação para a Ciência e a Tecnologia, under the project PEst-C/MAT/UI0324/2013).

I. Mozo Carollo gratefully acknowledges financial assistance from a Predoctoral Fellowship of the Basque Country Government (BFI-2012-262).

${ }^{*}$ Corresponding author

Email addresses: javier.gutierrezgarcia@ehu.eus (Javier Gutiérrez García), imanol.mozo@ehu.eus (Imanol Mozo Carollo), picado@mat.uc.pt (Jorge Picado)

URL: http://www.ehu.eus/javiergutierrezgarcia/ (Javier Gutiérrez García), http://www.mat.uc.pt/ picado (Jorge Picado)
} 
groups or many other familiar algebraic structures: if $S$ is a set of generators, $R$ is a set of relations $u=v$, where $u$ and $v$ are expressions in terms of the frame operations starting from elements and subsets of $S$, then there exists a frame $\operatorname{Frm}\langle S \mid R\rangle$ such that for any frame $L$, the set of frame homomorphisms $\operatorname{Frm}\langle S \mid R\rangle \rightarrow L$ is in a bijective correspondence with functions $f: S \rightarrow L$ that turn all relations in $R$ into identities in $L$ (see [22] for more details and some basic examples).

This is a very useful tool that allows, for instance, to define products in the category of locales with a construction that closely parallels the construction of the Tychonoff topology on a product space $[7,13]$, with advantage to the localic side (see [14]), or to define the frame of reals [16] just from the rationals, independently of any notion of real number, adding a new way of introducing the reals to the familiar classical ones (see [2]). For more examples see, for instance, the presentations of the Vietoris locale of a locale [15], the exponentials of locally compact locales [11], the Yosida locale of an abelian lattice-ordered group [18], the frame of complex numbers $[4,5]$, the frames of upper reals and lower reals [8], the frame of extended reals [3], the frame of partial reals [19] and the assembly of a frame [17].

Our aim with this paper is to settle the following question posed to us by Bernhard Banaschewski in a private communication:

Any idea how the topology of the unit circle fits in with frame presentations by generators and relations?

We provide two equivalent alternative presentations of the frame $\mathfrak{L}(\mathbb{T})$ of the unit circle. The first is the point-free counterpart of the Alexandroff compactification of the real line while the second is motivated by the standard construction of the unit circle space as the quotient space $\mathbb{R} / \mathbb{Z}$. Then, we lift the group operations of the frame of reals to the new frame $\mathfrak{L}(\mathbb{T})$, endowing it with a localic group structure.

The paper is organized as follows. We begin with a brief account of the necessary background and terminology (Section 2). In Section 3 we carry the construction of the Alexandroff extension of a frame. We then apply it to the particular case of the frame of reals (Section 4), obtaining this way a first presentation of the frame $\mathfrak{L}(\mathbb{T})$. In Section 5 we present an alternative set of generators and relations. In Section 6 we provide general criteria for concluding that an equalizer $e: E \rightarrow L$ of a pair $(f, g): L \rightarrow M$ of frame isomorphisms on a localic group $L$ lifts the group structure from $L$ into $E$. We then use these results, in the last section, to obtain the group structure of $\mathfrak{L}(\mathbb{T})$ induced by the canonical one in $\mathfrak{L}(\mathbb{R})$.

\section{Background}

Frames. A frame (or locale) $L$ is a complete lattice such that

$$
a \wedge \bigvee B=\bigvee\{a \wedge b \mid b \in B\}
$$


for all $a \in L$ and $B \subseteq L$; equivalently, it is a complete Heyting algebra with Heyting operation $\rightarrow$ satisfying the standard equivalence $a \wedge b \leq c$ iff $a \leq b \rightarrow c$. The pseudocomplement of $a \in L$ is the element

$$
a^{*}=a \rightarrow 0=\bigvee\{b \in L \mid a \wedge b=0\} .
$$

A frame homomorphism is a map $h: L \rightarrow M$ between frames which preserves finitary meets (including the top element 1 ) and arbitrary joins (including the bottom element 0 ). We denote by Frm the category of frames and frame homomorphisms. The category of locales, denoted by Loc, is the dual category of Frm.

An $S \subseteq L$ is a subframe of $L$ if it is closed under arbitrary joins and finite meets (in particular, $0,1 \in S$ ). Note that for each $a \in L, \uparrow a=\{x \in L \mid x \geq a\}$ and $\downarrow a=\{x \in L \mid x \leq a\}$ are frames (but, of course, not subframes of $L$ ).

The most typical example of a frame is the lattice $\mathfrak{O} X$ of open subsets of a topological space $X$. The correspondence $X \mapsto \mathfrak{O} X$ is clearly functorial (by taking inverse images), and consequently one has a contravariant functor $\mathfrak{O}:$ Top $\rightarrow$ Frm where Top is the category of topological spaces and continuous maps. There is also a functor in the opposite direction, the spectrum functor $\Sigma:$ Frm $\rightarrow$ Top, which assigns to each frame $L$ its spectrum $\Sigma L$, the space of all homomorphisms $\xi: L \rightarrow\{0,1\}$ with open sets $\Sigma_{a}=\{\xi \in \Sigma L \mid \xi(a)=1\}$ for any $a \in L$, and to each frame homomorphism $h: L \rightarrow M$ the continuous map $\Sigma h: \Sigma M \rightarrow \Sigma L$ such that $\Sigma h(\xi)=\xi \cdot h$. The spectrum functor is right adjoint to $\mathfrak{O}$, with adjunction maps

$$
\begin{aligned}
& \eta_{L}: L \rightarrow \mathfrak{O} \Sigma L, a \mapsto \Sigma_{a} \text { and } \\
& \varepsilon_{X}: X \rightarrow \Sigma \mathfrak{O} X, x \mapsto \widehat{x} \text { (given by } \widehat{x}(U)=1 \text { iff } x \in U \text { ). }
\end{aligned}
$$

The former is the spatial reflection of the frame $L$ and the latter is the sobrification of the space $X$.

For general notions and results concerning frames and locales we refer to Johnstone [13] or the more recent Picado-Pultr [20]. Here we recall a number of basic notions needed in the paper.

A frame $L$ is

- regular if $a=\bigvee\{b \in L \mid b \prec a\}$ for every $a \in L$, where $b \prec a$ ( $b$ is rather below a) means that $b^{*} \vee a=1$;

- completely regular if $a=\bigvee\{b \in L \mid b \prec \prec a\}$ for every $a \in L$, where $b \prec \prec a(b$ is completely below a) means that there is $\left\{c_{r} \mid r \in \mathbb{Q} \cap[0,1]\right\} \subseteq L$ such that $b \leq c_{0}, c_{1} \leq a$ and $c_{r} \prec c_{s}$ whenever $r<s$;

- compact if for each $A \subseteq L$ such that $\bigvee A=1$ there exists a finite $F \subseteq A$ such that $\bigvee F=1$;

- continuous if $a=\bigvee\{b \in L \mid b \ll a\}$ for every $a \in L$, where $b \ll a$ (b is way below a) means that $a \leq \bigvee A$ for some $A \subseteq L$ implies $b \leq \bigvee F$ for some finite $F \subseteq A$. 
A frame homomorphism $h: L \rightarrow M$ is

- dense if $h(a)=0$ implies $a=0$;

- a quotient map if it is onto.

Of course that one-to-one frame homomorphisms are dense. On the other hand, any dense frame homomorphism between regular frames with compact codomain is one-to-one.

Each frame homomorphism $h: L \rightarrow M$ preserves arbitrary joins and thus has a right adjoint $h_{*}: M \rightarrow L$ given by the equivalence

$$
h(a) \leq b \quad \text { iff } \quad a \leq h_{*}(b)
$$

for all $a \in L$ and $b \in M$. Specifically, $h_{*}(b)=\bigvee\{a \in L \mid h(a) \leq b\}$ for every $b \in M$.

Given a pair of frame homomorphisms $f, g: L \rightarrow M$, the embedding $e: E \subseteq$ $L$, where $E$ is the subframe $\{x \in L \mid f(x)=g(x)\}$, is the equalizer of $f$ and $g$ in Frm. This means that for any frame homomorphism $h: N \rightarrow L$ such that $f \cdot h=g \cdot h$ there exists a unique $\bar{h}: N \rightarrow E$ such that $e \cdot \bar{h}=h$ (evidently, $\bar{h}$ is given by $\bar{h}(x)=h(x)$ for every $x \in N)$.

Compactifications of frames. Given a frame $L$, a compactification of $L$ is an onto dense frame homomorphism $h: M \rightarrow L$ with a compact regular domain $M$. A frame is called compactifiable if it has a compactification. The set of all compactifications of $L$ is preordered by the relation $\left(h_{1}: M_{1} \rightarrow L\right) \leq\left(h_{2}: M_{2} \rightarrow\right.$ $L)$ iff there exists a frame homomorphism $g: M_{1} \rightarrow M_{2}$ such that $h_{2} \cdot g=h_{1}$. We denote by $\mathrm{K}(L)$ the corresponding poset induced by the usual identification of equivalent elements. We need to recall the familiar description of $\mathrm{K}(L)$ in terms of certain binary relations on $L$, due to Banaschewski [1].

A strong inclusion [1] on a frame $L$ is a binary relation $\triangleleft$ on $L$ such that

(1) If $x \leq a \triangleleft b \leq y$ then $x \triangleleft y$.

(2) $\triangleleft$ is a sublattice of $L \times L$.

(3) If $a \triangleleft b$ then $a \prec b$.

(4) If $a \triangleleft b$ then $a \triangleleft c \triangleleft b$ for some $c \in L$.

(5) If $a \triangleleft b$ then $b^{*} \triangleleft a^{*}$.

(6) $a=\bigvee\{b \in L \mid b \triangleleft a\}$ for all $a \in L$.

An ideal $J$ of $L$ is called a strongly regular $\triangleleft$-ideal if for any $x \in J$ there exists an $y \in J$ such that $x \triangleleft y$. The strongly regular $\triangleleft$-ideals of $L$ form a regular subframe of the frame $\mathfrak{I}(L)$ of all ideals of $L$, that we denote by

$$
\mathfrak{G}_{\triangleleft}(L) .
$$

Let $\mathrm{S}(L)$ be the set of all strong inclusions on the frame $L$, partially ordered as subsets of $L \times L$. By Proposition 2 of [1], $\mathrm{K}(L)$ is isomorphic to $\mathrm{S}(L)$. The isomorphism is given as follows: each compactification $h: M \rightarrow L$ of $L$ induces a strong inclusion $\triangleleft$ given by $x \triangleleft y$ iff $h_{*}(x) \prec h_{*}(y)$; conversely, given a 
strong inclusion relation $\triangleleft$ on $L$, the map $\mathfrak{G}_{\triangleleft}(L) \rightarrow L$ given by $I \mapsto \bigvee I$ is a compactification of $L$.

Moreover, a frame $L$ has a least compactification if and only if it is regular and continuous. In this case, the least compactification is given by the frame homomorphism $\bigvee: \mathfrak{G}_{\sqsubseteq}(L) \rightarrow L$, where $\sqsubseteq$ denotes the strong inclusion defined by

$$
a \sqsubseteq b \quad \text { iff } \quad a \prec b \text { and either } \uparrow\left(a^{*}\right) \text { or } \uparrow b \text { is compact. }
$$

Coproducts of frames. The coproduct $L \oplus M$ of two frames may be constructed in the following simple way. First take the Cartesian product $L \times M$ with the usual partial order and

$$
\mathfrak{D}(L \times M)=\{U \subseteq L \times M \mid \downarrow U=U \neq \emptyset\} .
$$

Call a $U \in \mathfrak{D}(L \times M)$ saturated if

(1) for any subset $A \subseteq L$ and any $b \in M$, if $A \times\{b\} \subseteq U$ then $(\bigvee A, b) \in U$, and

(2) for any $a \in L$ and any subset $B \subseteq M$, if $\{a\} \times B \subseteq U$ then $(a, \bigvee B) \in U$.

The set $A$ (resp. $B$ ) can be void; hence, in particular, each saturated set contains the set $\mathbb{O}=\{(0, b),(a, 0) \mid a \in L, b \in M\}$. It is easy to see that for each $(a, b) \in L \times M$,

$$
a \oplus b=\downarrow(a, b) \cup \mathbb{O} \text { is saturated. }
$$

To finish the construction take

$$
L \oplus M=\{U \in \mathfrak{D}(L \times M) \mid U \text { is saturated }\}
$$

with the coproduct injections

$$
\iota_{L}=(a \mapsto a \oplus 1): L \rightarrow L \oplus M, \quad \iota_{M}=(b \mapsto 1 \oplus b): M \rightarrow L \oplus M .
$$

Note that one has for each saturated $U$,

$$
U=\bigvee\{a \oplus b \mid(a, b) \in U\}=\bigcup\{a \oplus b \mid(a, b) \in U\},
$$

and if $a \oplus b \leq c \oplus d$ and $b \neq 0$ then $a \leq c$.

Localic groups. We recall that a localic group [12] is a group in the category of locales, i.e. a cogroup in Frm. Specifically, it is a frame $L$ endowed with three frame homomorphisms

$$
\mu: L \rightarrow L \oplus L, \quad \gamma: L \rightarrow L, \quad \varepsilon: L \rightarrow \mathbf{2}=\{0,1\}
$$

satisfying

$$
\begin{aligned}
& \left(\mu \oplus 1_{L}\right) \cdot \mu=\left(1_{L} \oplus \mu\right) \cdot \mu, \\
& \left(\varepsilon \oplus 1_{L}\right) \cdot \mu=\left(1_{L} \oplus \varepsilon\right) \cdot \mu=1_{L}, \quad \text { and } \\
& \nabla \cdot\left(\gamma \oplus 1_{L}\right) \cdot \mu=\nabla \cdot\left(1_{L} \oplus \gamma\right) \cdot \mu=\sigma_{L} \cdot \varepsilon
\end{aligned}
$$


where $\sigma_{L}: \mathbf{2} \rightarrow L$ sends 0 to 0 and 1 to 1 , and $\nabla$ is the codiagonal homomorphism $L \oplus L \rightarrow L$. A localic group $L$ is abelian when $\lambda \cdot \mu=\mu$ for the automorphism $\lambda: L \oplus L \rightarrow L \oplus L$ interchanging the two coproduct maps $L \rightarrow L \oplus L$.

Here, as usual, we make the (obvious) assumption that the cartesian products in the construction of coproducts are associative and we will work with the factor $\mathbf{2}$ as a void one, meaning that $L \oplus \mathbf{2}=\mathbf{2} \oplus L=L$ with coproduct injections

$$
L \stackrel{1_{L}}{\longrightarrow} L \stackrel{\sigma}{\longleftarrow} \mathbf{2} \text { and } \mathbf{2} \stackrel{\sigma}{\longrightarrow} L \stackrel{1_{L}}{\longleftarrow} L .
$$

The morphisms of localic groups (usually called $L G$-homomorphisms)

$$
h:(L, \mu, \gamma, \varepsilon) \rightarrow\left(L^{\prime}, \mu^{\prime}, \gamma^{\prime}, \varepsilon^{\prime}\right)
$$

are frame homomorphisms $h: L \rightarrow L^{\prime}$ such that

$$
\mu^{\prime} \cdot h=(h \oplus h) \cdot \mu, \quad \gamma^{\prime} \cdot h=h \cdot \gamma \quad \text { and } \quad \varepsilon^{\prime} \cdot h=\varepsilon .
$$

The dual of the resulting category is the category of localic groups. See [20] or [21] for more information on localic groups.

\section{The Alexandroff extension of a frame}

We shall say that an element $a$ of a frame $L$ is cocompact provided the frame $\uparrow a$ is compact. In the sequel, $\operatorname{coK}(L)$ denotes the set of all cocompact elements of $L$.

Remarks 3.1. (1) In case $L=\mathfrak{O} X$ for some space $X$, then $U \in \mathfrak{O} X$ is cocompact iff $X \backslash U$ is compact. This justifies our terminology.

(2) $a \in L$ is cocompact if and only if for each $B \subseteq L$ such that $a \vee(\bigvee B)=1$ there exists a finite $F \subseteq B$ such that $a \vee(\bigvee F)=1$. Indeed, let $a$ be cocompact and let $B \subseteq L$ satisfy $a \vee(\bigvee B)=1$. Then $\{a \vee b \mid b \in B\}$ is a cover of $\uparrow a$ and therefore there is a finite $F \subseteq B$ such that $1=\bigvee_{b \in F}(a \vee b)=a \vee(\bigvee F)$. For sufficiency, let $B$ be a cover of $\uparrow a$. Then $a \vee(\bigvee B)=1$ and thus there exists a finite $F \subseteq B$ such that $1=a \vee(\bigvee F)=\bigvee F$.

(3) $\operatorname{coK}(L)$ is a filter of $L$. Indeed:

(i) $1 \in \operatorname{coK}(L)$. ( $\uparrow 1=\{1\}$ is obviously compact).

(ii) If $a \in \operatorname{coK}(L)$ and $a \leq b$, then $b \in \operatorname{coK}(L)$ (since $\uparrow b \subseteq \uparrow a$ and $\bigvee \uparrow b=\bigvee \uparrow a$ ). Consequently, $\operatorname{coK}(L)$ is closed under non-void joins.

(iii) If $a_{1}, a_{2} \in \operatorname{coK}(L)$ then $a_{1} \wedge a_{2} \in \operatorname{coK}(L)$. In fact:

Let $B$ be a cover of $\uparrow\left(a_{1} \wedge a_{2}\right)$. Then, for $i=1,2,\left\{a_{i} \vee b \mid b \in B\right\}$ is a cover of $\uparrow a_{i}$ and so there exists a finite $F_{i} \subseteq B$ such that $a_{i} \vee\left(\bigvee F_{i}\right)=1$. Hence $1=\left(a_{1} \vee\left(\bigvee F_{1}\right)\right) \wedge\left(a_{2} \vee\left(\bigvee F_{2}\right)\right) \leq\left(a_{1} \wedge a_{2}\right) \vee \bigvee\left(F_{1} \cup F_{2}\right)=\bigvee\left(F_{1} \cup F_{2}\right)$, which shows that $F_{1} \cup F_{2}$ is a finite subcover of $\uparrow\left(a_{1} \wedge a_{2}\right)$.

(4) $\operatorname{coK}(L)=L$ if and only if 0 is cocompact if and only if $L$ is compact.

(5) The strong inclusion introduced in (2.2) can be equivalently stated as $a \sqsubseteq b$ iff $a \prec b$ and either $a^{*}$ or $b$ is cocompact. 
Proposition 3.2. For each continuous regular frame $L$ we have:

(1) If $a \ll 1$ then $a^{*} \in \operatorname{coK}(L)$.

(2) If $a \ll b$ then $a \sqsubseteq b$.

(3) For every $b \in \operatorname{coK}(L)$, there exists $c \in \operatorname{coK}(L)$ such that $c \prec b$.

(4) If there is some $b \in \operatorname{coK}(L)$ such that $b \ll 1$, then $L$ is compact.

Proof. (1) Let $B \subseteq L$ such that $a^{*} \vee(\bigvee B)=1$. Since $L$ is continuous, there exists $b \in L$ such that $a \ll b \ll 1$. Therefore, there exists a finite $F \subseteq B$ such that $b \leq a^{*} \vee(\bigvee F)$. Since $L$ is also regular, $a \ll b$ implies $a \prec b$ and we conclude that $1=a^{*} \vee b \leq a^{*} \vee(\bigvee F)$

(2) Since $L$ is regular, it follows immediately from (1) and Remark 3.1 (5).

(3) Let $b \in \operatorname{coK}(L)$. Since $L$ is continuous one has $1=\bigvee\{a \in L \mid a \ll 1\}$. Thus there exists some finite $F \subseteq\{a \in L \mid a \ll 1\}$ such that $b \vee(\bigvee F)=1$. Then, by (1), $a^{*} \in \operatorname{coK}(L)$ for every $a \in F$ and therefore $c=(\bigvee F)^{*}=\bigwedge_{a \in F} a^{*} \in \operatorname{coK}(L)$ since it is a finite meet of cocompact elements. Finally, $c^{*} \vee b \geq(\bigvee F) \vee b=1$.

(4) Let $b \in \operatorname{coK}(L)$ such that $b \ll 1$ and consider $A \subseteq L$ satisfying $\bigvee A=1$ Then there exists a finite $F_{1} \subseteq A$ such that $b \vee\left(\bigvee F_{1}\right)=1$ and a finite $F_{2} \subseteq A$ such that $b \leq \bigvee F_{2}$. Thus there exists a finite $F=F_{1} \cup F_{2}$ such that $\bigvee F=$ $\left(\bigvee F_{2}\right) \vee\left(\bigvee F_{1}\right) \geq b \vee\left(\bigvee F_{1}\right)=1$.

Now, given a frame $L$, consider the poset

$$
\mathscr{A}(L)=(L \times\{0\}) \cup(\operatorname{coK}(L) \times\{1\}) \subseteq L \times \mathbf{2}
$$

(endowed with the componentwise order). It is easy to check that $\mathscr{A}(L)$ is a frame. Indeed, it is a subframe of $L \times \mathbf{2}$, as it is closed under all suprema and finite infima (from the fact that $\operatorname{coK}(L)$ is a filter). In particular, for $A=\left(A_{0} \times\{0\}\right) \cup\left(A_{1} \times\{1\}\right) \subseteq \mathscr{A}(L)$, one has

$$
\bigvee A= \begin{cases}\left(\bigvee\left(A_{0} \cup A_{1}\right), 0\right) & \text { if } A_{1}=\varnothing, \\ \left(\bigvee\left(A_{0} \cup A_{1}\right), 1\right) & \text { if } A_{1} \neq \varnothing .\end{cases}
$$

We refer to $\mathscr{A}(L)$ as the Alexandroff extension of $L$.

Remarks 3.3. (1) This construction is a particular case of a general procedure introduced by Hong in [10] concerning extensions of a frame $L$ determined by a set of filters. Specifically, $\mathscr{A}(L)$ is the simple extension of $L$ with respect to a single filter, namely, $\operatorname{coK}(L)$.

(2) In the case that $L$ is the frame $\mathcal{O} X$ of open sets of a topological space $X$, $\mathscr{A}(L)$ is isomorphic to the lattice of open sets of the Alexandroff extension of $X$.

Proposition 3.4. $\mathscr{A}(L)$ is a compact frame. 
Proof. Let $A \subseteq \mathscr{A}(L)$ such that $\bigvee A=\left(1_{L}, 1\right)$. Then $\bigvee\left(A_{0} \cup A_{1}\right)=1_{L}$ and there exists some $b \in \operatorname{coK}(L)$ such that $(b, 1) \in A$. Consequently, there exists a finite $F \subseteq A_{0} \cup A_{1}$ such that $b \vee(\bigvee F)=1_{L}$. It follows that for the finite subset

$$
B=\left(\left(F \cap A_{0}\right) \times\{0\}\right) \cup\left(\left(F \cap A_{1}\right) \times\{1\}\right) \cup\{(b, 1)\} \subseteq A
$$

one has $\bigvee B=(b \vee(\bigvee F), 1)=\left(1_{L}, 1\right)$

Recall (2.1) and (2.2).

Proposition 3.5. Let $L$ be a non-compact continuous regular frame. The map $f: \mathfrak{G}_{\sqsubseteq}(L) \rightarrow \mathscr{A}(L)$ given by

$$
f(I)= \begin{cases}(\bigvee I, 0) & \text { if } I \cap \operatorname{coK}(L)=\varnothing, \\ (\bigvee I, 1) & \text { otherwise, }\end{cases}
$$

is a frame isomorphism with inverse $g: \mathscr{A}(L) \rightarrow \mathfrak{G}_{\sqsubseteq}(L)$ given by

$$
g(a, 0)=\{x \in L \mid x \ll a\} \quad \text { and } \quad g(b, 1)=\{x \in L \mid x \prec b\}
$$

for every $a \in L$ and $b \in \operatorname{coK}(L)$.

Proof. Consider the map $\varphi: \mathfrak{G}_{\sqsubseteq}(L) \rightarrow \mathbf{2}$ defined by

$$
\varphi(I)= \begin{cases}0 & \text { if } I \cap \operatorname{coK}(L)=\varnothing, \\ 1 & \text { otherwise }\end{cases}
$$

It is easy to check that $\varphi$ is a frame homomorphism. Putting $\varphi$ together with the frame homomorphism $\bigvee: \mathfrak{G}_{\sqsubseteq}(L) \rightarrow L$, we get the frame homomorphism

$$
f: \mathfrak{G}_{\sqsubseteq}(L) \rightarrow L \times \mathbf{2}
$$

given by $I \mapsto(\bigvee I, \varphi(I))$. Obviously, $f\left(\mathfrak{G}_{\sqsubseteq}(L)\right) \subseteq \mathscr{A}(L)$. As an abuse of notation, we shall consider $\mathscr{A}(L)$ as the codomain of $f$. Since $f$ is dense, $\mathscr{A}(L)$ is compact and $\mathfrak{G}_{\sqsubseteq}(L)$ is regular, we conclude that $f$ is one-to-one.

The subsets $g(a, 0)=\{x \in L \mid x \ll a\}$ and $g(b, 1)=\{x \in L \mid x \prec b\}$ are obviously ideals of $L$ for any $a \in L$ and $b \in \operatorname{coK}(L)$. On the other hand, given $a \in L$ and $x \in g(a, 0)$, by the continuity of $L$ there exists $y \in L$ such that $x \ll y \ll a$. Since $L$ is regular, $y \in g(a, 0)$ and, by Proposition 3.2(2), we conclude that $x \sqsubseteq y$. Thus $g(a, 0)$ is a strongly regular $\sqsubseteq$-ideal. Further, given $b \in \operatorname{coK}(L)$ and $x \in g(b, 1)$, one has that $x \sqsubseteq b$. Then there exists $y \in L$ such that $x \sqsubseteq y \sqsubseteq b$ and so $y \in g(b, 1)$. It follows that $g(b, 1)$ is a strongly regular 巨-ideal.

Moreover, we know by Proposition $3.2(3)$ that $g(b, 1) \cap \operatorname{coK}(L) \neq \varnothing$ for every $b \in \operatorname{coK}(L)$ and thus $f(g(b, 1))=(\bigvee g(b, 1), 1)$. Finally, since $L$ is non-compact, it follows from Proposition 3.2 (4) that $g(a, 0) \cap \operatorname{coK}(L)=\varnothing$ for every $a \in L$ and so $f(g(a, 0))=(\bigvee g(a, 0), 0)$. Accordingly, since $L$ is regular and continuous, we conclude that $f \cdot g=1_{\mathscr{A}(L)}$, and thus $f$ is onto.

This shows that for non-compact continuous regular frames, the first projection $\pi_{1}: \mathscr{A}(L) \rightarrow L$ defined by $\pi_{1}(a, 0)=a$ and $\pi_{1}(b, 1)=b$ is the least compactification of $L$. We call it the Alexandroff compactification of $L$. 


\section{The Alexandroff compactification of $\mathfrak{L}(\mathbb{R})$}

Let $\mathfrak{L}(\mathbb{R})$ denote the frame of reals [2], that is, the frame generated by all ordered pairs $(p, q)$ of rationals, subject to the relations

(R1) $(p, q) \wedge(r, s)=(p \vee r, q \wedge s)$,

(R2) $(p, q) \vee(r, s)=(p, s)$ whenever $p \leq r<q \leq s$,

(R3) $(p, q)=\bigvee\{(r, s) \mid p<r<s<q\}$,

(R4) $\bigvee\{(p, q) \mid p, q \in \mathbb{Q}\}=1$.

Further, let $(p,-)=\bigvee_{q>p}(p, q)$ and $(-, q)=\bigvee_{p<q}(p, q)$ for each $p, q \in \mathbb{Q}$. Note that

$$
(p, q)^{*}=(-, p) \vee(q,-), \quad(p,-)^{*}=(-, p) \quad \text { and } \quad(-, q)^{*}=(q,-) .
$$

Remarks 4.1. (1) The assignment

$$
(p, q) \mapsto\langle p, q\rangle \equiv\{t \in \mathbb{Q} \mid p<t<q\}
$$

for every $p, q \in \mathbb{Q}$ determines a canonical quotient frame homomorphism (see $[2$, p. 10])

$$
h: \mathfrak{L}(\mathbb{R}) \rightarrow \mathfrak{O Q},
$$

since it is onto and it trivially turns the defining relations $(\mathrm{R} 1)-(\mathrm{R} 4)$ of $\mathfrak{L}(\mathbb{R})$ into identities in $\mathfrak{O} \mathbb{Q}$. Of course $h$ is not one-to-one: e.g.,

$$
h\left(\bigvee\left\{(-, q) \mid q^{2}<2\right\} \vee \bigvee\left\{(p,-) \mid p^{2}>2 \text { and } p>0\right\}\right)=\mathbb{Q}=h(1)
$$

Nevertheless, it is a dense map. Indeed, since $\{(p, q) \mid p, q \in \mathbb{Q}\}$ is a join-basis of $\mathfrak{L}(\mathbb{R})$, it is enough to prove that $h((p, q))=\varnothing$ implies $(p, q)=0$, but this is easy since $\langle p, q\rangle=\varnothing$ implies that $p \geq q$ and by (R3) it follows that $(p, q)=0$. In particular, $(p, q)=0$ if and only if $p \geq q$ in $\mathbb{Q}$.

$(2) \mathfrak{L}(\mathbb{R})$ is a continuous and regular frame [2]. Indeed, one has that $(r, s) \ll$ $(p, q)$, and in consequence also $(r, s) \prec(p, q)$, whenever $p<r<s<q$. Accordingly, the least compactification of $\mathfrak{L}(\mathbb{R})$ does exist.

It is well known that $(p, q)^{*}$ is a cocompact element of $\mathfrak{L}(\mathbb{R})$ for any $p, q \in \mathbb{Q}$ (since the frame $\uparrow((-, p) \vee(q,-))$ is compact for any $p, q \in \mathbb{Q}$, see [2]). We characterize the cocompact elements of $\mathfrak{L}(\mathbb{R})$ as follows:

Proposition 4.2. The following are equivalent for each $a \in \mathfrak{L}(\mathbb{R})$ :

(1) $a$ is cocompact.

(2) There exist $p, q \in \mathbb{Q}$ such that $(p, q)^{*} \leq a$.

(3) There exist $p, q \in \mathbb{Q}$ such that $(p, q) \vee a=1$.

Proof. (3) $\Longrightarrow(2)$ is obvious and (2) $\Longrightarrow(1)$ follows from Remark 3.1 (3). Finally, if $a$ is cocompact then, since $a \vee \bigvee\{(p, q) \mid p, q \in \mathbb{Q}\}=1$, there exists $\left\{\left(p_{i}, q_{i}\right)\right\}_{i=1}^{n}$ such that $a \vee\left(\bigvee_{i=1}^{n}\left(p_{i}, q_{i}\right)\right)=1$. Consequently, $a \vee(p, q)=1$ for $p=\min _{i=1}^{n} p_{i}$ and $q=\max _{i=1}^{n} q_{i}$. 
Since any element of $\mathfrak{L}(\mathbb{R})$ is a join of basic generators $(p, q)$ (by relation $(\mathrm{R} 1))$, we have the following characterization:

Corollary 4.3. An element a of $\mathfrak{L}(\mathbb{R})$ is cocompact if and only if there exist $p, q \in \mathbb{Q}$ and $\left\{p_{i}, q_{i}\right\}_{i \in I} \subseteq \mathbb{Q}$ such that

$$
a=(p, q)^{*} \vee \bigvee_{i \in I}\left(p_{i}, q_{i}\right)
$$

Consequently, in $\mathscr{A}(\mathfrak{L}(\mathbb{R}))$ any element is a join of elements of the form

$$
((p, q), 0) \quad \text { and } \quad\left((p, q)^{*}, 1\right) \quad(p, q \in \mathbb{Q}) .
$$

As we will show in detail, this yields an equivalent description of $\mathscr{A}(\mathfrak{L}(\mathbb{R}))$, in terms of generators and relations, with the elements

$$
(p, q) \equiv((p, q), 0)
$$

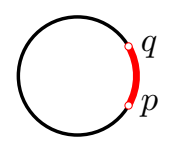

and $\overparen{p, q} \equiv\left((p, q)^{*}, 1\right)$

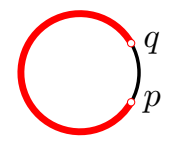

as basic generators.

Let $\mathfrak{A}(\mathbb{R})$ be the frame presented by generators $(p, q)$ and $\overparen{p, q}$, with $p, q \in \mathbb{Q}$, and subject to the following relations:

(R1) $(p, q) \wedge(r, s)=(p \vee r, q \wedge s)$,

(R2) $(p, q) \vee(r, s)=(p, s)$ whenever $p \leq r<q \leq s$,

(R3) $(p, q)=\bigvee\{(r, s) \mid p<r<s<q\}$,

(S1) $\overparen{p, q} \wedge \overparen{r, s}=\overparen{p, s}$ whenever $p \leq r \leq q \leq s$,

(S2) $\overparen{p, q} \vee \overparen{r, s}=\overparen{p \vee r, q \wedge s}$,

(S3) $\overparen{p, q}=\bigvee\{\overparen{r, s} \mid r<p$ and $q<s\}$,

$(\mathrm{S} 4)(p, q) \wedge \overparen{r, s}=(p, q \wedge r) \vee(p \vee s, q)$,

(S5) $(p, q) \vee \overparen{r}, s=1$ whenever $p<r$ and $s<q$.

We have:

\section{Lemma 4.4.}

(1) If $p \geq q$ then $(p, q)=0$.

(2) If $p \leq r$ and $s \leq q$ then $\overparen{p, q} \leq \overparen{r, s}$.

(3) If $q \leq r$ or $s \leq p$ then $(p, q) \leq \overparen{r, s}$.

(4) If $p<r \leq q \leq s$ then $(p, q) \vee \overparen{r, s}=\overparen{q, s}$.

(5) If $r \leq p \leq s<q$ then $(p, q) \vee \overparen{r, s}=\overparen{r}, p$.

(6) If $p \leq q<r \leq s$ then $\overparen{p, q} \wedge \overparen{r, s}=\overparen{p, s} \vee(q, r)$.

(7) If $p>q$ then $\overparen{p, q}=1$.

(8) If $p<r<s<q$ then $(r, s) \prec \prec(p, q)$ and $\overparen{r, s} \prec \prec \overparen{p, q}$. 
Proof. (1) Apply (R3).

(2) Apply (S3).

(3) If $q \leq r$ then, by (S4), $(p, q) \wedge \overparen{r, s}=(p, q) \vee(p \vee s, q)=(p, q)$. Similarly, if $s \leq p$ then $(p, q) \wedge \overparen{r, s}=(p, q \wedge r) \vee(p, q)=(p, q)$

(4) Let $p, q, r, s \in \mathbb{Q}$ such that $p<r \leq q \leq s$. Since $p<r$ and $s<s+1$ it follows by $(\mathrm{S} 5)$ that $(p, s+1) \vee \widetilde{r, s}=1$. Then, by $(\mathrm{R} 2),(p, q) \vee(r, s+1) \vee \overparen{r, s}=1$. Hence, by (S1), (S4) and (3),

$$
\begin{aligned}
\overparen{q, s} & =\overparen{q, s} \wedge((p, q) \vee(r, s+1) \vee \overparen{r, s}) \\
& =(\overparen{q, s} \wedge(p, q)) \vee(\overparen{q, s} \wedge(r, s+1)) \vee(\overparen{q, s} \wedge \overparen{r, s}) \\
& =(p, q) \vee(r, q) \vee(s, s+1) \vee \overparen{r, s}=(p, q) \vee \overparen{r, s} .
\end{aligned}
$$

(5) Similar to (4).

(6) If $p \leq q<r \leq s$, then, by properties (4) and (5) and (R1), one has

$$
\begin{aligned}
\overparen{p, q} \wedge \overparen{r, s} & =((q, s+1) \vee \overparen{p, s}) \wedge((p-1, r) \vee \overparen{p, s}) \\
& =((q, s+1) \wedge(p-1, r)) \vee \overparen{p, s}=(q, r) \vee \overparen{p, s} .
\end{aligned}
$$

(7) Let $r \in \mathbb{Q}$ such that $q<r<p$. By (S5), $\overparen{p, q}=(r, r) \vee \overparen{p, q}=1$.

(8) First note that $(p, q) \wedge \overparen{p, q}=0$ for every $p<q$ in $\mathbb{Q}$. Indeed, by (S4), $(p, q) \wedge \overparen{p, q}=(p, p) \vee(q, q)$ which is 0 by (R3). Therefore $(p, q) \leq \overparen{p, q}^{*}$ and $\overparen{p, q} \leq(p, q)^{*}$. Then, by (S5), for every $p<r<s<q$ in $\mathbb{Q}$ we have

$$
(r, s)^{*} \vee(p, q) \geq \overparen{r, s} \vee(p, q)=1 \quad \text { and } \quad \widetilde{r, s}^{*} \vee \overparen{p, q} \geq(r, s) \vee \overparen{p, q}=1
$$

Hence $(r, s) \prec(p, q)$ and $\overparen{r, s} \prec \overparen{p, q}$. From this it follows readily that

$$
(r, s) \prec\left(\frac{p+r}{2}, \frac{q+s}{2}\right) \prec(p, q) \quad \text { and } \quad \overparen{r, s} \prec \overparen{\frac{p+r}{2}, \frac{q+s}{2}} \prec \overparen{p, q} .
$$

This interpolation can be repeated indefinitely and we get $(r, s) \prec \prec(p, q)$ and $\overparen{r, s} \prec \prec \overparen{p, q}$.

Combining Lemma 4.4 with (R3) and (S3), we obtain immediately the following:

Proposition 4.5. $\mathfrak{A}(\mathbb{R})$ is completely regular.

Further, we have:

Lemma 4.6. The set of generators of $\mathfrak{A}(\mathbb{R})$ forms a join-basis. 
Proof. We only need to check that finite meets of generators are expressible as joins of generators. By $(\mathrm{R} 1)$ and $(\mathrm{S} 4),(p, q) \wedge(r, s)$ and $(p, q) \wedge \overparen{r, s}$ are obviously joins of generators. So it remains to check the case $\overparen{p, q} \wedge \overparen{r, s}$. We may assume that $p \leq q$ and $r \leq s$ since the other cases are straightforward, by Lemma $4.4(7)$. Further, we may assume without loss of generality that $p \leq r$. If $p \leq r \leq q \leq s$ we are done by (S1). If $p \leq r \leq s<q$, then $\overparen{p, q} \wedge \overparen{r, s}=\overparen{p, q}$, by Lemma 4.4 (2). Finally, the case $p \leq q<r \leq s$ follows from Lemma 4.4(6).

Theorem 4.7. The assignments

$$
(p, q) \mapsto((p, q), 0) \quad \text { and } \quad \overparen{p, q} \mapsto\left((p, q)^{*}, 1\right)
$$

determine a frame isomorphism $\Psi: \mathfrak{A}(\mathbb{R}) \rightarrow \mathscr{A}(\mathfrak{L}(\mathbb{R}))$.

Proof. In order to show that $\Psi$ is a frame homomorphism it suffices to check that it turns the defining relations (R1)-(R3) and (S1)-(S5) into identities in the frame $\mathscr{A}(\mathfrak{L}(\mathbb{R}))$. Of course, it turns (R1)-(R3) into identities trivially, so we only have to check it for relations (S1)-(S5).

(S1) Let $p \leq r \leq q \leq s$ in $\mathbb{Q}$. Then

$$
\begin{aligned}
\Psi(\overparen{p, q}) \wedge \Psi(\overparen{r, s}) & =\left((p, q)^{*} \wedge(r, s)^{*}, 1\right) \\
& =(((-, p) \vee(q,-)) \wedge((-, r) \vee(s,-)), 1) \\
& =((-, p \wedge r) \vee(s, p) \vee(q, r) \vee(s \vee q,-), 1) \\
& =((-, p) \vee(s,-), 1)=\left((p, s)^{*}, 1\right)=\Psi(\overparen{p, s})
\end{aligned}
$$

(S2) Let $p, q, r, s \in \mathbb{Q}$. Then

$$
\begin{aligned}
\Psi(\overparen{p, q}) \vee \Psi(\overparen{r, s}) & =\left((p, q)^{*} \vee(r, s)^{*}, 1\right)=((-, p) \vee(q,-) \vee(-, r) \vee(s,-), 1) \\
& =((-, p \vee r) \vee(q \wedge s,-), 1)=\left((p \vee r, q \wedge s)^{*}, 1\right) \\
& =\Psi(\stackrel{p \vee r, q \wedge s}{ }) .
\end{aligned}
$$

(S3) Let $p, q \in \mathbb{Q}$. Then

$$
\begin{aligned}
\Psi(\overparen{p, q}) & =\left((p, q)^{*}, 1\right)=((-, p) \vee(q,-), 1)=\left(\bigvee_{r<p}(-, r) \vee \bigvee_{s>q}(s,-), 1\right) \\
& =\bigvee\left\{\left((r, s)^{*}, 1\right) \mid r<p \text { and } q<s\right\}=\bigvee\{\Psi(\overparen{r, s}) \mid r<p \text { and } q<s\} .
\end{aligned}
$$

(S4) Let $p, q, r, s \in \mathbb{Q}$. Then

$$
\begin{aligned}
\Psi(p, q) \wedge \Psi(\overparen{r, s}) & =((p, q), 0) \wedge\left((r, s)^{*}, 1\right)=((p, q) \wedge((-, r) \vee(s,-)), 0) \\
& =((p, q \wedge r) \vee(p \vee s, q), 0)=\Psi(p, q \wedge r) \vee \Psi(p \vee s, q) .
\end{aligned}
$$

(S5) If $p<r$ and $s<q$ in $\mathbb{Q}$, then

$$
\begin{aligned}
\Psi(p, q) \vee \Psi(\overparen{r, s}) & =((p, q), 0) \vee\left((r, s)^{*}, 1\right)=((p, q) \vee(-, r) \vee(s,-), 1) \\
& =(((p,-) \vee(-, r) \vee(s,-)) \wedge((-, q) \vee(-, r) \vee(s,-)), 1) \\
& =(((p \wedge s,-) \vee(-, r)) \wedge((-, q \vee r) \vee(s,-)), 1)=1
\end{aligned}
$$


since $p \wedge s<r$ and $s<q \vee r$.

Moreover, by Corollary $4.3, \Psi$ is obviously onto. In order to verify that $\Psi$ is also one-to-one, we only have to check that it is dense, since $\mathscr{A}(\mathfrak{L}(\mathbb{R}))$ is a compact regular frame and $\mathfrak{A}(\mathbb{R})$ is regular. First, note that $\Psi(\overparen{p, q}) \neq(0,0)$ for any $p, q \in \mathbb{Q}$. Furthermore, $\Psi(p, q)=(0,0)$ implies that $(p, q)=0$ in $\mathfrak{L}(\mathbb{R})$. Consequently, $p \geq q$ by Remark 4.1. Then, $(p, q)=0$ in $\mathfrak{A}(\mathbb{R})$, by (R3). The conclusion now follows from Lemma 4.6.

Summarizing, since $\mathfrak{L}(\mathbb{R})$ is a non-compact continuous regular frame [2], $\mathfrak{A}(\mathbb{R})$ is its Alexandroff compactification.

\section{An alternative presentation for $\mathscr{A}(\mathfrak{L}(\mathbb{R}))$ : the frame of the unit circle}

In this section we provide an equivalent presentation for $\mathscr{A}(\mathfrak{L}(\mathbb{R}))$. The motivation for it comes from the description of the unit circle space as a quotient of $\mathbb{R}$.

Definition 5.1. The frame of the unit circle is the frame $\mathfrak{L}(\mathbb{T})$ generated by all ordered pairs $(p, q)$, for $p, q \in \mathbb{Q}$, subject to the defining relations

(T1) $(p, q) \wedge(r, s)=(p \vee r, q \wedge s)$ whenever $q \vee s-p \wedge r \leq 1$,

(T2) $(p, q) \vee(r, s)=(p, s)$ whenever $p \leq r<q \leq s$,

(T3) $(p, q)=\bigvee\{(r, s) \mid p<r<s<q\}$,

(T4) $\bigvee_{p, q \in \mathbb{Q}}(p, q)=1$,

(T5) $(p, q)=(p+1, q+1)$.

Remarks 5.2. (1) If $p \geq q$ then $(p, q)=0$, by (T3).

(2) If $q-p>1$ then $(p, q)=1$. Indeed, by (T5), (T2) and (T3) one has

$$
\begin{aligned}
(p, q) & =\bigvee_{m=0}^{n+1}(p+m, q+m)=(p, q+n+1)=(p-\lfloor p\rfloor-1, q+n-\lfloor p\rfloor) \\
& \geq(0, n)
\end{aligned}
$$

for every $n \in \mathbb{N}$. Given $r, s \in \mathbb{Q}$, (T5) and (T3) ensure that

$$
(r, s)=(r-\lfloor r\rfloor, s-\lfloor r\rfloor) \leq(0,\lfloor s\rfloor-\lfloor r\rfloor+1) \leq(p, q) .
$$

Hence $(p, q) \geq \bigvee_{r, s \in \mathbb{Q}}(r, s)=1$ by $(\mathrm{T} 4)$.

(3) For any $p, q \in \mathbb{Q}$ satisfying $0<q-p \leq 1$ one has $(p, q)=(r, s)$ for some $0 \leq r<1$ and $r<s \leq r+1$ (just take $r=p-\lfloor p\rfloor$ and $s=q-\lfloor p\rfloor$ ).

(4) Comparing (T1) with (R1) one notice some restriction on $p, q, r, s$. The reason for it is that with no such restriction in (T1) we would have, for any $p, q \in \mathbb{Q}$ satisfying $q-p \leq 1,(p, q)=(p, q) \wedge(p+1, q+1)=(p+1, q)$, which is 0 by remark (1). This would lead ultimately to the unwanted fact $\mathfrak{L}(\mathbb{T})=\{0=1\}$ ! 
Accordingly, $\mathfrak{L}(\mathbb{T})$ is not isomorphic to the quotient $\mathfrak{L}(\mathbb{R})$ modulo the congruence generated by the pair $((p, q),(p+1, q+1))$.

(5) If $p<r<s<q$ then $(r, s) \prec \prec(p, q)$. Indeed, if $q-p>1$, then the result follows immediately from remark (2) above. On the other hand, if $q-p \leq 1$, then it follows from (T1) that $(r, s) \wedge(s-1, r)=0$. Therefore $(s-1, r) \leq(r, s)^{*}$ and consequently $(r, s)^{*} \vee(p, q) \geq(s-1, r) \vee(p, q)=(s-1, q)=1$, by (T2) and remark (2). Hence $(r, s) \prec(p, q)$. From this it follows that

$$
(r, s) \prec\left(\frac{p+r}{2}, \frac{q+s}{2}\right) \prec(p, q)
$$

and since this interpolation can be continued indefinitely we conclude that $(r, s) \prec \prec(p, q)$.

Combining Remark $5.2(5)$ with (T3), we obtain immediately the following:

Proposition 5.3. $\mathfrak{L}(\mathbb{T})$ is completely regular.

Next we establish the precise relation between $\mathfrak{L}(\mathbb{T})$ and the usual space $\mathbb{T}$ of the unit circle.

Proposition 5.4. The spectrum of $\mathfrak{L}(\mathbb{T})$ is homeomorphic to the space $[0,1\rangle$ endowed with the topology generated by the family of sets $\langle p, q\rangle$ and $[0, p\rangle \cup\langle q, 1\rangle$ for every $p<q$ in $\mathbb{Q} \cap\langle 0,1\rangle$.

Proof. For each $x \in[0,1\rangle$ let $h_{x}: \mathfrak{L}(\mathbb{T}) \rightarrow \mathbf{2}$ be given by

$$
h_{x}(p, q)=1 \quad \text { iff } \quad x \in\langle p-\lfloor p\rfloor, q-\lfloor p\rfloor\rangle \cup\langle p-\lfloor p\rfloor-1, q-\lfloor p\rfloor-1\rangle .
$$

It is easy to show that $h_{x}$ turns the defining relations (R1)-(R5) into identities and so $h_{x} \in \Sigma \mathfrak{L}(\mathbb{T})$. Let $\rho:[0,1\rangle \rightarrow \Sigma \mathfrak{L}(\mathbb{T})$ be given by $\rho(x)=h_{x}$. In order to show that $\rho$ is one-to-one, let $x_{1} \neq x_{2}$ in $[0,1\rangle$. If, say, $x_{1}<x_{2}$, there exist $p, q \in \mathbb{Q}$ such that $x_{1}<p<x_{2}<q<1$ and then $h_{x_{1}}(p, q)=0$ and $h_{x_{2}}(p, q)=1$ and so $h_{x_{1}} \neq h_{x_{2}}$.

The function $\rho$ is also onto. Indeed, given $h \in \Sigma \mathfrak{L}(\mathbb{T})$, we distinguish two cases:

(i) If $h((0,1))=0$ then, by (R2),

$$
h((p, q))=h((0,1) \vee(p-\lfloor p\rfloor, q-\lfloor p\rfloor))=h((0,(q-\lfloor p\rfloor) \vee 1))
$$

for every $p, q \in \mathbb{Q}$ and so

$$
\begin{aligned}
h((p, q))=1 & \Longleftrightarrow q-\lfloor p\rfloor>1 \Longleftrightarrow 0 \in\langle p-\lfloor p\rfloor-1, q-\lfloor p\rfloor-1\rangle \\
& \Longleftrightarrow 0 \in\langle p-\lfloor p\rfloor, q-\lfloor p\rfloor\rangle \cup\langle p-\lfloor p\rfloor-1, q-\lfloor p\rfloor-1\rangle \\
& \Longleftrightarrow h_{0}((p, q))=1 .
\end{aligned}
$$

Hence $h=h_{0}=\rho(0)$.

(ii) If $h((0,1))=1$ then, by (R3) and the compactness of $\mathbf{2}$, there exist $p_{0}, q_{0} \in$ $\mathbb{Q}$ such that $0<p_{0}<q_{0}<1$ and $h\left(\left(p_{0}, q_{0}\right)\right)=1$. Then

$$
0<\bigvee\{p \in\langle 0,1\rangle \cap \mathbb{Q} \mid h((p, 1))=1\}=\bigwedge\{q \in\langle 0,1\rangle \cap \mathbb{Q} \mid h((0, q))=1\}<1
$$


Let

$$
x_{h}=\bigvee\{p \in\langle 0,1\rangle \cap \mathbb{Q} \mid h((p, 1))=1\}=\bigwedge\{q \in\langle 0,1\rangle \cap \mathbb{Q} \mid h((0, q))=1\}
$$

Then

$$
h((p, q))=1 \Longleftrightarrow x_{h} \in\langle p-\lfloor p\rfloor, q-\lfloor p\rfloor\rangle \cup\langle p-\lfloor p\rfloor-1, q-\lfloor p\rfloor-1\rangle
$$

and therefore $h=h_{x_{h}}=\rho\left(x_{h}\right)$.

It remains to show that $\rho$ is a homeomorphism. For each open set $\Sigma_{(p, q)}$ of $\Sigma \mathfrak{L}(\mathbb{T})$

$$
\begin{aligned}
\rho^{-1}\left(\Sigma_{(p, q)}\right) & =\left\{x \in[0,1\rangle \mid h_{x} \in \Sigma_{(p, q)}\right\}=\left\{x \in[0,1\rangle \mid h_{x}((p, q))=1\right\} \\
& =\{x \in[0,1\rangle \mid x \in\langle p-\lfloor p\rfloor, q-\lfloor p\rfloor\rangle \cup\langle p-\lfloor p\rfloor-1, q-\lfloor p\rfloor-1\rangle\} \\
& =[0, q-\lfloor p\rfloor-1\rangle \cup\langle p-\lfloor p\rfloor,(q-\lfloor p\rfloor) \wedge 1\rangle .
\end{aligned}
$$

Hence $\rho$ is continuous. On the other hand, for each $p, q \in \mathbb{Q}$ such that $0<p<$ $q<1$,

$$
\rho(\langle p, q\rangle)=\left\{h_{x} \in \Sigma \mathfrak{L}(\mathbb{T}) \mid p<x<q\right\}=\{h \in \Sigma \mathfrak{L}(\mathbb{T}) \mid h(p, q)=1\}=\Sigma_{(p, q)}
$$

and

$$
\begin{aligned}
\rho([0, p\rangle \cup\langle q, 1\rangle) & =\left\{h_{x} \in \Sigma \mathfrak{L}(\mathbb{T}) \mid 0 \leq x<p \text { or } q<x<1\right\} \\
& =\{h \in \Sigma \mathfrak{L}(\mathbb{T}) \mid h(q, p+1)=1\}=\Sigma_{(q, p+1)}
\end{aligned}
$$

are open sets of $\Sigma \mathfrak{L}(\mathbb{T})$.

Corollary 5.5. The spectrum of $\mathfrak{L}(\mathbb{T})$ is homeomorphic to the unit circle $\mathbb{T}$.

Remark 5.6. It should be added that the homeomorphism $\rho:[0,1\rangle \rightarrow \Sigma \mathfrak{L}(\mathbb{T})$ induces a frame isomorphism $\mathfrak{O} \Sigma \mathfrak{L}(\mathbb{T}) \rightarrow \mathfrak{O}([0,1\rangle)$ taking $\Sigma_{(p, q)}$ to the interval $\langle p, q\rangle$, as seen in the proof above of Proposition 5.4. Combining this with the definition of the spatial reflection of a frame $L$, we conclude that the frame homomorphism $\mathfrak{L}(\mathbb{T}) \rightarrow \mathfrak{O}(\mathbb{T})$ taking $(p, q)$ to $\langle p, q\rangle$ is the spatial reflection map.

Finally, we investigate the relation between the frames $\mathfrak{L}(\mathbb{T})$ and $\mathfrak{A}(\mathbb{R})$.

Proposition 5.7. Let $\varphi: \mathbb{Q} \rightarrow\langle 0,1\rangle \cap \mathbb{Q}$ be an order isomorphism. The map $\Phi: \mathfrak{A}(\mathbb{R}) \rightarrow \mathfrak{L}(\mathbb{T})$ defined by

$$
(p, q) \mapsto(\varphi(p), \varphi(q)) \quad \text { and } \quad \overparen{p, q} \mapsto(\varphi(q), \varphi(p)+1)
$$

for all $p, q \in \mathbb{Q}$ is an onto frame homomorphism.

Proof. In order to show that $\Phi$ is a frame homomorphism we only need to check that $\Phi$ turns the defining relations (R1)-(R3) and (S1)-(S5) of $\mathscr{A}(\mathfrak{L}(\mathbb{R})$ ) into identities in $\mathfrak{L}(\mathbb{T})$. We first note that Remarks $5.2(1)$ and (2) imply that $\Phi(p, q)=0$ whenever $q \leq p$ and that $\Phi(\widetilde{p, q})=1$ whenever $q<p$. 
(R1) follows directly from (T1) since $\varphi(p)-\varphi(q) \leq 1$ for all $p, q \in \mathbb{Q}$.

(R2) and (R3) follow directly from (T2) and (T3), respectively.

(S1) Let $p \leq r \leq q \leq s$ in $\mathbb{Q}$. Then, by (T1),

$$
\begin{aligned}
\Phi(\overparen{p, q}) & \wedge \Phi(\overparen{r, s})=(\varphi(q), \varphi(p)+1) \wedge(\varphi(s), \varphi(r)+1) \\
& =(\varphi(q) \vee \varphi(s), \varphi(p) \wedge \varphi(r)+1)=(\varphi(s), \varphi(p)+1)=\Phi(\overparen{p, s}),
\end{aligned}
$$

since $((\varphi(p)+1) \vee(\varphi(r)+1))-(\varphi(q) \wedge \varphi(s))=\varphi(r)+1-\varphi(q) \leq 1$ as $\varphi(r) \leq \varphi(q)$.

(S2) Let $p, q, r, s \in \mathbb{Q}$. Then

$$
\begin{aligned}
\Phi(\overparen{p, q}) & \vee \Phi(\overparen{r, s})=(\varphi(q), \varphi(p)+1) \vee(\varphi(s), \varphi(r)+1) \\
& =(\varphi(q) \wedge \varphi(s), \varphi(p)+1) \vee(\varphi(s), \varphi(p) \vee \varphi(r)+1) \\
& =(\varphi(q) \wedge \varphi(s), \varphi(p) \vee \varphi(r)+1)=\Phi(\overparen{p \vee r, q \wedge s}),
\end{aligned}
$$

(by (T3) and (T2) since $\varphi(q) \wedge \varphi(s) \leq \varphi(s)<\varphi(p)+1 \leq \varphi(p) \vee \varphi(r)+1$ ).

(S3) Let $p, q \in \mathbb{Q}$. Since $\varphi$ is an order isomorphism, then, by (T3),

$$
\begin{aligned}
\bigvee\{\Phi(\overparen{r, s}) \mid r<p \text { and } q<s\}=\bigvee\{(\varphi(s), \varphi(r)+1) \mid r<p \text { and } q<s\} \\
\quad=\bigvee\{(\varphi(s), \varphi(r)+1) \mid \varphi(q)<\varphi(s)<\varphi(r)+1<\varphi(p)+1\} \\
=(\varphi(q), \varphi(p)+1)=\Phi(\overparen{p, q}) .
\end{aligned}
$$

(S4) Let $p, q, r, s \in \mathbb{Q}$. We distinguish several cases:

(i) If $q \leq p$ or $r \leq p<q \leq s$ then, by (T1),

$$
\Phi(p, q) \wedge \Phi(\overparen{r, s})=0=\Phi(p, q \wedge r) \vee \Phi(p \vee s, q) .
$$

(ii) If $s<r, p<q \leq r \leq s$ or $r \leq s \leq p<q$ then, by (T2) and (T3),

$$
\Phi(p, q) \wedge \Phi(\overparen{r, s})=(\varphi(p), \varphi(q))=\Phi(p, q \wedge r) \vee \Phi(p \vee s, q)
$$

(iii) If $r \leq p \leq s<q$ then by (R1),

$$
\Phi(p, q) \wedge \Phi(\overparen{r, s})=(\varphi(s), \varphi(q))=\Phi(p, q \wedge r) \vee \Phi(p \vee s, q) .
$$

(iv) If $p<r \leq q \leq s$ then by (R1) and (R5),

$$
\Phi(p, q) \wedge \Phi(\overparen{r, s})=(\varphi(p), \varphi(r))=\Phi(p, q \wedge r) \vee \Phi(p \vee s, q)
$$

(v) If $p<r \leq s<q$ then by (iv) and (v),

$$
\Phi(p, q) \wedge \Phi(\overparen{r, s})=(\varphi(p), \varphi(r)) \vee(\varphi(s), \varphi(q))=\Phi(p, q \wedge r) \vee \Phi(p \vee s, q)
$$


(S5) Let $p<r$ and $s<q$ in $\mathbb{Q}$. If $s \leq p$ then $s<r$ and so $\Phi(\overparen{r, s})=1$. Otherwise, if $p<s$ then $\varphi(p)<\varphi(s)<\varphi(q)<\varphi(r)+1$ and using (T2),

$$
\begin{aligned}
\Phi(p, q) & \vee \Phi(\overparen{r, s})=(\varphi(p), \varphi(q)) \vee(\varphi(s), \varphi(r)+1)=(\varphi(p), \varphi(r)+1) \\
& =\Phi(\overparen{r, p})=1 .
\end{aligned}
$$

In all, this shows that $\Phi$ is actually a frame homomorphism. The ontoness of $\Phi$ follows from Remark $5.2(3)$. Indeed, given $p, q \in \mathbb{Q}$ such that $0 \leq p<1$ and $p<q \leq p+1$ one has

$$
\begin{aligned}
& \Phi\left(\varphi^{-1}(p), \varphi^{-1}(q)\right)=(p, q) \quad \text { if } q \leq 1 \quad \text { and } \\
& \Phi(\overbrace{\varphi^{-1}(q-1), \varphi^{-1}(p)})=(p, q) \quad \text { if } q>1 .
\end{aligned}
$$

Corollary 5.8. The set of generators of $\mathfrak{L}(\mathbb{T})$ forms a join-basis.

Proof. This is an immediate consequence of Lemma 4.6 and the fact that the set of generators of $\mathfrak{A}(\mathbb{R})$ is mapped by $\Phi$ onto the set of generators of $\mathfrak{L}(\mathbb{T})$.

Remark 5.9. Of course, the ontoness of $\Phi$ also gives an alternative proof of the fact that $\mathfrak{L}(\mathbb{T})$ is a completely regular frame, since $\mathfrak{A}(\mathbb{R})$ is completely regular (Proposition 4.5).

Proposition 5.10. Let $f: \mathfrak{L}(\mathbb{R}) \rightarrow \mathfrak{L}(\mathbb{R})$ be the frame isomorphism given by $(p, q) \mapsto(p+1, q+1)$ for all $p, q \in \mathbb{Q}$. The equalizer of the pair $\left(f, 1_{\mathfrak{L}(\mathbb{R})}\right)$ is the map e: $\mathfrak{L}(\mathbb{T}) \rightarrow \mathfrak{L}(\mathbb{R})$ defined by

$$
(p, q) \mapsto \bigvee_{n \in \mathbb{Z}}(p+n, q+n)
$$

Proof. Obviously, $f$ is a frame isomorphism with inverse $f^{-1}$ given by $(p, q) \mapsto$ $(p-1, q-1)$ for each $p, q \in \mathbb{Q}$. In order to prove that $e$ is a frame homomorphism, we will check that it turns defining relations $(\mathrm{T} 1)-(\mathrm{T} 5)$ into identities in $\mathfrak{L}(\mathbb{R})$ :

We note that if $q-p \leq 0$ then $e(p, q)=0$ and if $q-p>1$ then $p+n<p+n+$ $1<q+n<q+n+1$ for every $n \in \mathbb{Z}$ and thus $e(p, q)=\bigvee_{n \in \mathbb{N}}(p+n, q+n)=1$ by repeated application of (R2).

(T1) Let $p, q, r, s \in \mathbb{Q}$ such that $q \vee s-p \wedge r \leq 1$. Then

$$
q+n \leq r+n+1 \leq r+m
$$

for each $m>n$ in $\mathbb{Z}$ and

$$
s+m \leq p+m+1 \leq p+n
$$


for each $m<n$ in $\mathbb{Z}$ and so $(p+n, q+n) \wedge(r+m, s+m)=0$ for every $m \neq n$ in $\mathbb{Z}$. Hence

$$
\begin{aligned}
e(p, q) \wedge e(r, s) & =\left(\bigvee_{n \in \mathbb{Z}}(p+n, q+n)\right) \wedge\left(\bigvee_{m \in \mathbb{N}}(r+m, s+m)\right) \\
& =\bigvee_{n, m \in \mathbb{Z}}((p+n, q+n) \wedge(r+m, s+m)) \\
& =\bigvee_{n \in \mathbb{Z}}((p+n, q+n) \wedge(r+n, s+n)) \\
& =\bigvee_{n \in \mathbb{Z}}((p \vee r)+n,(q \wedge s)+n)=e(p \vee r, q \wedge s) .
\end{aligned}
$$

(T2) Let $p, q, r, s \in \mathbb{Q}$ such that $p \leq r<q \leq s$. It is easy to check that $e(p, q) \vee e(r, s) \leq e(p, s)$. On the other hand

$$
\begin{aligned}
e(p, q) & \vee e(r, s)=\bigvee_{n \in \mathbb{Z}}(p+n, q+n) \vee \bigvee_{m \in \mathbb{N}}(r+m, s+m) \\
& \geq \bigvee_{n \in \mathbb{Z}}((p+n, q+n) \vee(r+n, s+n))=\bigvee_{n \in \mathbb{Z}}(p+n, s+n)=e(p, s) .
\end{aligned}
$$

(T3) Let $p, q \in \mathbb{Q}$. Then

$$
\begin{aligned}
\bigvee_{n \in \mathbb{Z}} e(p, q) & =\bigvee_{n \in \mathbb{Z}}(p+n, q+n)=\bigvee_{n \in \mathbb{Z} p+n<r<s<q+n}(r, s) \\
& =\bigvee_{n \in \mathbb{Z}} \bigvee_{p<r<s<q}(r+n, s+n)=\bigvee_{p<r<s<q} e(r, s) .
\end{aligned}
$$

(T4) $\bigvee_{p, q \in \mathbb{Q}} e(p, q) \geq e(0,2)=\bigvee_{n \in \mathbb{Z}}(n, n+2)=1$

(T5) Let $p, q \in \mathbb{Q}$. Then

$$
e(p, q)=\bigvee_{n \in \mathbb{Z}}(p+n, q+n)=\bigvee_{n \in \mathbb{Z}}(p+n+1, q+n+1)=e(p+1, q+1)
$$

Now, let

$$
E=\{x \in \mathfrak{L}(\mathbb{R}) \mid f(x)=x\}
$$

be the equalizer of $f$ and $1_{\mathfrak{L}(\mathbb{R})}$. Obviously, by the definition of $e$, one has that $e(p, q) \in E$ for every $p, q \in \mathbb{Q}$. Since $\{(p, q)\}_{p, q \in \mathbb{Q}}$ generates $\mathfrak{L}(\mathbb{T})$, then $e(\mathfrak{L}(\mathbb{T})) \subseteq E$. On the other hand, if $x \in E$ and $p, q \in \mathbb{Q}$ are such that $(p, q) \leq x$ in $\mathfrak{L}(\mathbb{R})$ then $(p+1, q+1)=f(p, q) \leq f(x)=x$ and $f(p-1, q-1)=(p, q) \leq$ $x=f(x)$. Consequently, $(p-1, q-1) \leq x$ (since $f$ is an isomorphism). By induction, it follows that $(p+n, q+n) \leq x$ for every $n \in \mathbb{Z}$ and thus

$$
e(p, q)=\bigvee_{n \in \mathbb{Z}}(p+n, q+n) \leq x .
$$

Hence

$$
\begin{aligned}
x & =\bigvee\{(p, q) \mid(p, q) \leq x\}=\bigvee\left\{\bigvee_{n \in \mathbb{Z}}(p+n, q+n) \mid(p, q) \leq x\right\} \\
& =\bigvee\{e(p, q) \mid(p, q) \leq x\}=e(\bigvee\{(p, q) \mid(p, q) \leq x\})
\end{aligned}
$$


and therefore $e(x) \in e(\mathfrak{L}(\mathbb{T}))$. In conclusion, $e(\mathfrak{L}(\mathbb{T}))=E$. It suffices now to show that $e$ is one-to-one. Let

$$
h: \mathfrak{L}(\mathbb{R}) \rightarrow \uparrow((-, 0) \vee(1,-))
$$

be the frame homomorphism given by $x \mapsto x \vee(-, 0) \vee(1,-)$. For each $p, q \in \mathbb{Q}$ such that $0 \leq p<1$ and $p<q \leq p+1$ one has

$$
\begin{aligned}
(h \cdot e)(p, q) & =\bigvee_{n \in \mathbb{Z}}(p+n, q+n) \vee(-, 0) \vee(1,-) \\
& =(p-1, q-1) \vee(p, q) \vee(-, 0) \vee(1,-) \geq(p, q) \vee(-, 0) \vee(1,-) .
\end{aligned}
$$

Indeed:

- for each $n \geq 2,(p-n, q-n) \leq(p-n, 0) \leq(-, 0)$ by (R3);

- for each $n \geq 1,(p+n, q+n) \leq(1, q+n) \leq(1,-)$ also by (R3).

Moreover, $(h \cdot e)(p, q) \neq 0$ by Remark 4.1. Then we may conclude that $h \cdot e$ is dense by the fact that the set of generators of $\mathfrak{L}(\mathbb{T})$ is a join-basis combined with Remark $5.2(2)$. Since $\mathfrak{L}(\mathbb{T})$ is a regular frame and $\uparrow((-, 0) \vee(1,-))$ is regular and compact, it follows that $h \cdot e$, and hence $e$, is one-to-one.

From now on, when convenient, we will identify the frame of the unit circle with the complete sublattice $e(\mathfrak{L}(\mathbb{T}))$ of $\mathfrak{L}(\mathbb{R})$.

Corollary 5.11. $\mathfrak{L}(\mathbb{T})$ is compact.

Proof. This is now obvious since the frame homomorphism

$$
h \cdot e: \mathfrak{L}(\mathbb{T}) \rightarrow \uparrow((-, 0) \vee(1,-))
$$

is one-to-one and $\uparrow((-, 0) \vee(1,-))$ is a compact frame.

Corollary 5.12. $\mathfrak{L}(\mathbb{T})$ is spatial.

Proof. Classically, the exponential map exp: $\mathbb{R} \rightarrow \mathbb{T}, x \mapsto e^{2 \pi i x}$, may be described as the coequalizer ofthe pair of continuous functions $1_{\mathbb{R}}, \gamma: \mathbb{R} \rightarrow \mathbb{R}$ $(\gamma(x)=x-1)$. Since the contravariant functor $\mathfrak{O}:$ Top $\rightarrow$ Frm is a left adjoint, it turns colimits into limits. So one has the equalizer diagram

$$
\mathfrak{O}(\mathbb{T}) \stackrel{\mathfrak{O}(\exp )}{\longrightarrow} \mathfrak{O}(\mathbb{R}) \underset{\mathfrak{O}\left(1_{\mathbb{R}}\right)}{\stackrel{\mathfrak{O}(\gamma)}{\longrightarrow}} \mathfrak{O}(\mathbb{R})
$$

in Frm. It suffices now to combine the proposition with the well known result that $\mathfrak{L}(\mathbb{R})$ is isomorphic to $\mathfrak{O}(\mathbb{R})$ (we note that the proof of this result is constructively valid under the assumption that the closed intervals $[p, q]$ are compact, see [2, Remark 4]).

Of course, the spatiality of $\mathfrak{L}(\mathbb{T})$ follows immediately from Corollary 5.11, albeit with the Prime Ideal Theorem. 
Corollary 5.13. The frame homomorphism $\Phi$ from Proposition 5.7 is an isomorphism.

Proof. It remains to show that $\Phi$ is one-to-one. Since $\mathfrak{A}(\mathbb{R})$ is regular and $\mathfrak{L}(\mathbb{T})$ is both regular and compact, it suffices to check that $\Phi$ is a dense map. So let $p, q \in \mathbb{Q}$. Then $\Phi(\overparen{p, q})=(\varphi(q), \varphi(p)+1) \neq 0$. In fact, applying the equalizer $e$ of the proposition, we have $e(\Phi(\overparen{p, q}))=e(\varphi(q), \varphi(p)+1)$, which is non-zero by the canonical frame homomorphism $\mathfrak{L}(\mathbb{R}) \rightarrow \mathfrak{O}(\mathbb{Q})$ (recall Remark 4.1), since $\varphi(q)<1<\varphi(p)+1$. Similarly, $\Phi(p, q) \neq 0$ whenever $p<q$, i.e. $(p, q) \neq 0$. By Lemma 4.6 we conclude that $\Phi$ is dense.

Remark 5.14. It is a straightforward exercise to check that the inverse of $\Phi$ is given by

$$
\Phi^{-1}(p, q)= \begin{cases}0 & \text { if } q \leq p, \\ \overbrace{\varphi^{-1}(q-\lfloor p\rfloor-1), \varphi^{-1}(p-\lfloor p\rfloor)} & \text { if } p<\lfloor p\rfloor+1<q \leq p+1, \\ 1 & \text { if } q>p+1,\end{cases}
$$

for every $p, q \in \mathbb{Q}$. Applying Lemma 4.4, this simplifies to

$$
\Phi^{-1}(p, q)= \begin{cases}\left(\varphi^{-1}(p-\lfloor p\rfloor), \varphi^{-1}(q-\lfloor p\rfloor)\right) & \text { if } q \leq\lfloor p\rfloor+1, \\ \varphi^{-1}(q-\lfloor p\rfloor-1), \varphi^{-1}(p-\lfloor p\rfloor) & \text { if }\lfloor p\rfloor+1<q .\end{cases}
$$

Further, by Remark $5.2(2)$, this leads to

$$
\Phi^{-1}(p, q)= \begin{cases}\left(\varphi^{-1}(p), \varphi^{-1}(q)\right) & \text { if } q \leq 1, \\ \overbrace{\varphi^{-1}(q-1), \varphi^{-1}(p)} & \text { if } 1<q\end{cases}
$$

for all $p, q \in \mathbb{Q}$ and $0 \leq p<1$.

\section{Induced localic group structures}

In this section, we analyze when an equalizer like the one of Proposition 5.10 lifts the localic group structure from the codomain into the domain. This will be the crucial step in the description next section of the localic group structure of $\mathfrak{L}(\mathbb{T})$.

We begin by recalling that for any frame homomorphisms $f_{1}: L_{1} \rightarrow M_{1}$ and $f_{2}: L_{2} \rightarrow M_{2}$, the homomorphism $f_{1} \oplus f_{2}$ is the unique frame homomorphism $L_{1} \oplus L_{2} \rightarrow M_{1} \oplus M_{2}$ making the following diagram commute:

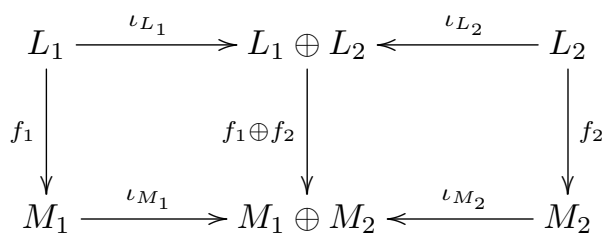


It is clear that $\left(f_{1} \oplus f_{2}\right)\left(\bigvee_{i \in I}\left(a_{i} \oplus b_{i}\right)\right)=\bigvee_{i \in I}\left(f_{1}\left(a_{i}\right) \oplus f_{2}\left(b_{i}\right)\right)$ and therefore compositions of morphisms of this type satisfy $\left(f_{1} \oplus f_{2}\right) \cdot\left(g_{1} \oplus g_{2}\right)=\left(f_{1} \cdot g_{1}\right) \oplus$ $\left(f_{2} \cdot g_{2}\right)$.

Our first lemma may well be known but since we have no reference for it we include its proof. In it $L$ and $M$ are frames, $E$ is a complete sublattice of $L$ and $e$ denotes the inclusion frame homomorphism $E \rightarrow L$. For each $(a, b) \in E \times M$, $a \oplus b$ and $a \bar{\oplus} b$ denote the corresponding basic generator of respectively $E \oplus M$ and $L \oplus M$.

Lemma 6.1. The frame homomorphism

$$
e \oplus 1_{M}: E \oplus M \rightarrow L \oplus M
$$

is given by $\left(e \oplus 1_{M}\right)(U)=\downarrow_{L \times M} U$ for each $U \in E \times M$. In particular, $e \oplus 1_{M}$ is one-to-one.

Proof. Let $U \in E \oplus M$. We first show that $\downarrow_{L \times M} U$ is actually an element of $L \oplus M$ :

(1) Let $A \subseteq L$ and $b \in M$ such that $A \times\{b\} \subseteq \downarrow_{L \times M} U$. Then for each $a \in A$ there exists $a^{\prime} \in E$ such that $a \leq a^{\prime}$ and $\left(a^{\prime}, b\right) \in U$. It follows that $\left(\bigvee\left\{a^{\prime} \mid a \in A\right\}, b\right) \in U$ and thus $(\bigvee A, b) \in \downarrow_{L \times M} U$.

(2) Let $a \in L$ and $B \subseteq M$ such that $\{a\} \times B \subseteq \downarrow_{L \times M} U$. Then for each $b \in B$ there exists $a_{b} \in E$ such that $a \leq a_{b}$ and $\left(a_{b}, b\right) \in U$. Let $a^{\prime}=\bigwedge_{b \in B}^{L} a_{b} \in E$. Clearly, $\left(a^{\prime}, b\right) \in U$ and $(a, b) \leq\left(a^{\prime}, b\right)$ for every $b \in B$. Hence $(a, \bigvee B) \leq$ $\left(a^{\prime}, \bigvee B\right) \in U$.

Note, moreover, that for each $(a, b) \in U$

$$
\left(e \oplus 1_{M}\right)(a \oplus b)=a \bar{\oplus} b=\downarrow_{L \times M}(a \oplus b) \subseteq \downarrow_{L \times M} U .
$$

Since

$$
U=\bigvee_{(a, b) \in U}^{E \oplus M}(a \oplus b)=\bigcup_{(a, b) \in U}(a \oplus b)
$$

then

$$
\left(e \oplus 1_{M}\right)(U)=\bigvee_{(a, b) \in U}^{L \oplus M}(a \bar{\oplus} b) \subseteq \downarrow_{L \times M} U .
$$

On the other hand it is clear that

$$
\downarrow_{L \times M} U \subseteq \bigcup_{(a, b) \in U}(a \bar{\oplus} b) \subseteq \bigvee_{(a, b) \in U}^{L \oplus M}(a \bar{\oplus} b) .
$$

Hence $\left(e \oplus 1_{M}\right)(U)=\downarrow_{L \times M} U$.

Remarks 6.2. (1) We can say a little more: $E \oplus M$ is isomorphic to the subframe of $L \oplus M$ generated by all $a \bar{\oplus} b, a \in E, b \in M$, since $\{a \oplus b\}_{(a, b) \in E \times M}$ generates $E \oplus M$ and $\left(e \oplus 1_{M}\right)(a \oplus b)=a \bar{\oplus} b$ for each $(a, b) \in E \times M$. In the following, we will make an abuse of notation and will regard $E \oplus M$ as that subframe of $L \oplus M$.

(2) We note in addition that, of course, applying Lemma 6.1 twice leads to the fact that $e \oplus e$ is a monomorphism. 
For the next two results, note that if $f, g: L \rightarrow N$ are complete lattice homomorphism then so is their equalizer $e: E \rightarrow L$, meaning that $E$ is a complete sublattice of $L$.

Lemma 6.3. Let $f, g: L \rightarrow N$ be frame isomorphisms with equalizer e: $E \rightarrow L$. For any frame $M$,

$$
E \oplus M \stackrel{e \oplus 1_{M}}{\longrightarrow} L \oplus M \stackrel{f \oplus 1_{M}}{\underset{g \oplus 1_{M}}{\stackrel{f}{~}}} N \oplus M
$$

is an equalizer diagram in Frm.

Proof. We know by the previous lemma that $E \oplus M$ may be regarded as the subframe of $L \oplus M$ generated by all $a \bar{\oplus} b, a \in E, b \in M$. It now suffices to show that this is precisely the subframe consisting of all $U \in L \oplus M$ such that $\left(f \oplus 1_{M}\right)(U)=\left(g \oplus 1_{M}\right)(U)$. Of course, $\left(f \oplus 1_{M}\right)(U)=\left(g \oplus 1_{M}\right)(U)$ for every $U \in E \oplus M$. Conversely, let $U \in L \oplus M$ such that $\left(f \oplus 1_{M}\right)(U)=\left(g \oplus 1_{M}\right)(U)$ and consider $a \in L$ and $b \in M$ such that $(a, b) \in U$. Furthermore, let

$$
a^{\prime}=\bigvee_{n \in \mathbb{Z}} h^{n}(a),
$$

where $h=g^{-1} \cdot f, h^{0}=1_{L}, h^{n}(n>0)$ denotes the composite $h \cdot h \cdots h$ ( $n$ times) and $h^{n}(n<0)$ denotes the composite $h^{-1} \cdot h^{-1} \cdots h^{-1}$ ( $-n$ times). Evidently, $a \leq a^{\prime}$. Moreover, $a^{\prime} \in E$. Indeed,

$$
g\left(a^{\prime}\right)=\bigvee_{n \in \mathbb{Z}} f\left(h^{n-1}(a)\right)=\bigvee_{n \in \mathbb{Z}} f\left(h^{n}(a)\right)=f\left(a^{\prime}\right) .
$$

Notice also that $(h(a), b) \in U$ since

$$
f(a) \oplus b \leq\left(f \oplus 1_{P}\right)(U)=\left(g \oplus 1_{P}\right)(U) \Longleftrightarrow\left(g^{-1} f\right)(a) \oplus b \leq U .
$$

Then, by symmetry, $\left(h^{-1}(a), b\right) \in U$. Proceeding inductively we eventually conclude that $\left(h^{n}(a), b\right) \in U$ for every $n \in \mathbb{Z}$ and thus $\left(a^{\prime}, b\right) \in U$. In summary, we have proved that for any $(a, b) \in U$ there is some $\left(a^{\prime}, b\right) \geq(a, b)$ still in $U$ with $a^{\prime} \in E$. This guarantees that $U \in E \oplus M$.

Proposition 6.4. Let

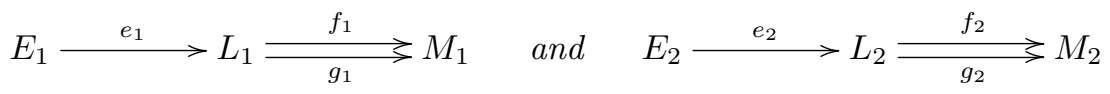

be equalizers in Frm with $f_{1}, g_{1}, f_{2}$ and $g_{2}$ frame isomorphisms. Then the homomorphism

$$
E_{1} \oplus E_{2} \stackrel{e_{1} \oplus e_{2}}{\longrightarrow} L_{1} \oplus L_{2}
$$


is the limit of the diagram

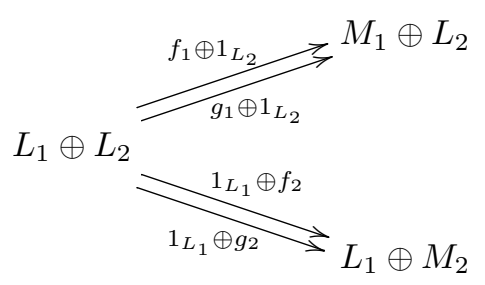

Proof. Let $h: N \rightarrow L_{1} \oplus L_{2}$ be a frame homomorphism such that

$$
\left(1_{L_{1}} \oplus f_{2}\right) \cdot h=\left(1_{L_{1}} \oplus g_{2}\right) \cdot h \quad \text { and } \quad\left(f_{1} \oplus 1_{L_{2}}\right) \cdot h=\left(g_{1} \oplus 1_{L_{2}}\right) \cdot h .
$$

By Lemma 6.3,

$$
L_{1} \oplus E_{2} \stackrel{1_{L_{1}} \oplus e_{2}}{\longrightarrow} L_{1} \oplus L_{2} \underset{1_{L_{1}} \oplus g_{2}}{\stackrel{1_{L_{1}} \oplus f_{2}}{\longrightarrow}} L_{1} \oplus M_{2}
$$

is an equalizer so there exists $h^{\prime}: N \rightarrow L_{1} \oplus E_{2}$ such that $\left(1_{L_{1}} \oplus e_{2}\right) \cdot h^{\prime}=$ $h$. We have then the following commutative diagram, where $1_{M_{1}} \oplus e_{2}$ is a monomorphism (by Lemma 6.1).

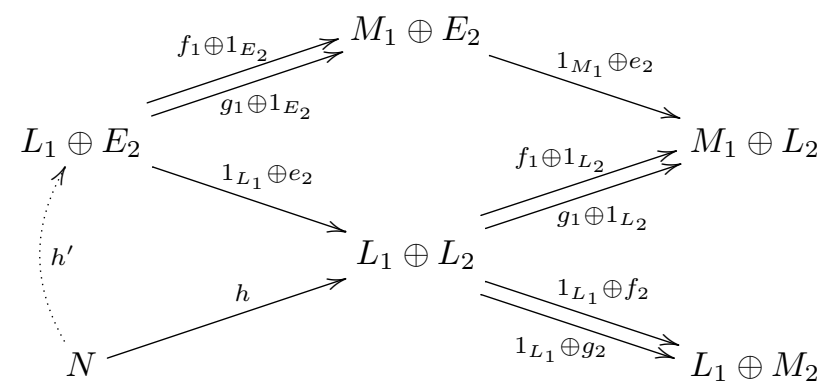

Then, immediately, $\left(f_{1} \oplus 1_{E_{2}}\right) \cdot h^{\prime}=\left(g_{1} \oplus 1_{E_{2}}\right) \cdot h^{\prime}$. Finally, since $e_{1} \oplus 1_{E_{2}}$ is the equalizer of $f_{1} \oplus 1_{E_{2}}$ and $g_{1} \oplus 1_{E_{2}}$ (again by Lemma 6.3), there is some $h^{\prime \prime}$ making the leftmost triangle in the following diagram

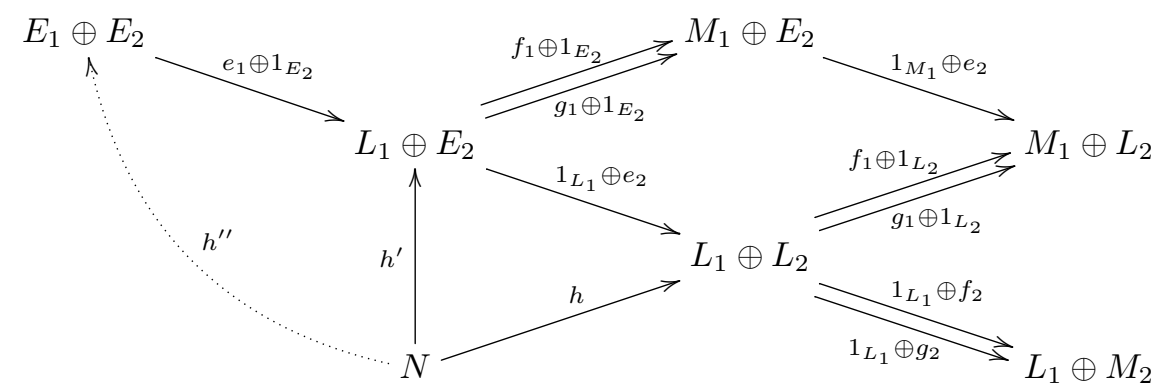

to commute. 
Now let $(L, \mu, \gamma, \varepsilon)$ be an arbitrary localic group and

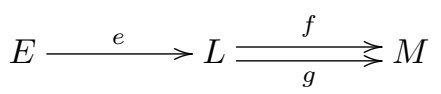

an equalizer where $f$ and $g$ are frame isomorphisms such that

$$
\left(f \oplus 1_{L}\right) \cdot \mu \cdot e=\left(g \oplus 1_{L}\right) \cdot \mu \cdot e, \quad\left(1_{L} \oplus f\right) \cdot \mu \cdot e=\left(1_{L} \oplus g\right) \cdot \mu \cdot e
$$

and

$$
f \cdot \gamma \cdot e=g \cdot \gamma \cdot e .
$$

Under these conditions, it is possible to lift the localic group structure of $L$ into $E$, in the following manner:

(LG1) (7.3.1) and Proposition 6.4 lead to an $\bar{\mu}: E \rightarrow E \oplus E$ satisfying $(e \oplus e) \cdot \bar{\mu}=$ $\mu \cdot e$.

(LG2) (7.3.2) and the fact that $e$ is the equalizer of $f$ and $g$ yield an $\bar{\gamma}: E \rightarrow E$ satisfying $e \cdot \bar{\gamma}=\gamma \cdot e$.

(LG3) $\bar{\varepsilon}: E \rightarrow \mathbf{2}$ is the composite $\varepsilon \cdot e$.

Remark 6.5. Note that $\bar{\varepsilon}$ may be defined alternatively using the equalizer. Indeed, since $f \cdot \sigma \cdot \varepsilon \cdot e=g \cdot \sigma \cdot \varepsilon \cdot e$, then the equalizer $e$ yields some $\varepsilon^{\prime}: E \rightarrow E$ such that $e \cdot \varepsilon^{\prime}=\sigma \cdot \varepsilon \cdot e$ but, as any frame homomorphism, it factors as

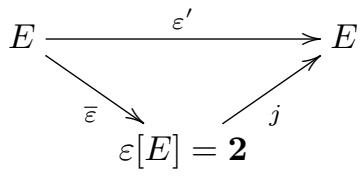

Theorem 6.6. $(E, \bar{\mu}, \bar{\gamma}, \bar{\varepsilon})$ is a localic group. If $L$ is abelian so is $E$.

Proof. It is just a matter of checking that conditions (LG1)-(LG3) allow to lift the commutativity of the diagrams in the definition of the localic group $(L, \mu, \gamma, \varepsilon)$ to the commutativity of the corresponding diagrams in $(E, \bar{\mu}, \bar{\gamma}, \bar{\varepsilon})$. For instance, regarding associativity of $\bar{\mu}$, that is, the commutativity of square $(A)$ in the next diagram

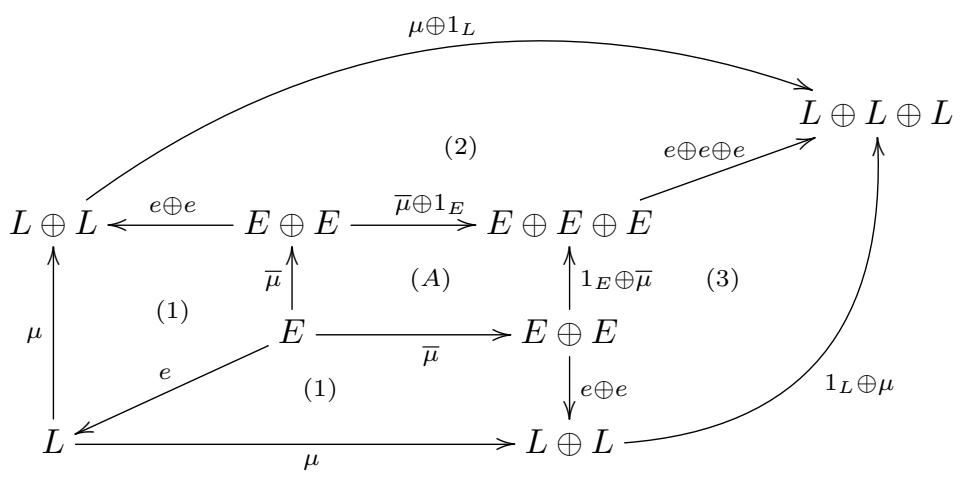


it follows immediately from the commutativity of the outing quadrilateral (which corresponds to the associativity of $\mu$ in $L$ ), the commutativity of subdiagrams (1), (2) and (3) (from (LG1)), and the fact that $e \oplus e \oplus e$ is a monomorphism (from Remark 6.2(2)).

The remaining properties may be checked in a similar way.

We shall refer to $(\bar{\mu}, \bar{\gamma}, \bar{\varepsilon})$ as the localic group structure on $E$ induced by $(L, \mu, \gamma, \varepsilon)$ and $e: E \rightarrow L$.

\section{The localic group structure of $\mathfrak{L}(\mathbb{T})$}

Now we are in the position to establish the localic group structure of $\mathfrak{L}(\mathbb{T})$. For this, we need to recall from [2, p. 39] some of the familiar lattice-ordered ring operations of $\mathfrak{L}(\mathbb{R})$ (see [9] for a detailed presentation):

(1) For each $r \in \mathbb{Q}$, the nullary operation $r: \mathfrak{L}(\mathbb{R}) \rightarrow \mathbf{2}=\{0,1\}$ given by

$$
\mathrm{r}(p, q)=1 \text { if and only if } r \in\langle p, q\rangle .
$$

(2) For each $\kappa>0$ in $\mathbb{Q}$, the unary operation $\omega_{\kappa}: \mathfrak{L}(\mathbb{R}) \rightarrow \mathfrak{L}(\mathbb{R})$, representing the scalar multiplication by $\kappa$, defined by

$$
\omega_{\kappa}(p, q)=\left(\frac{p}{\kappa}, \frac{q}{\kappa}\right) .
$$

Similarly, for each $\kappa<0$ in $\mathbb{Q}, \omega_{\kappa}$ is given by $\omega_{\kappa}(p, q)=\left(\frac{q}{\kappa}, \frac{p}{\kappa}\right)$.

(3) The binary operation $+: \mathfrak{L}(\mathbb{R}) \rightarrow \mathfrak{L}(\mathbb{R}) \oplus \mathfrak{L}(\mathbb{R})$ is defined by

$$
+(p, q)=\bigvee_{r \in \mathbb{Q}}\left(\left(r, r+\frac{q-p}{2}\right) \oplus\left(p-r, \frac{p+q}{2}-r\right)\right) .
$$

We denote the operations

$$
0: \mathfrak{L}(\mathbb{R}) \rightarrow \mathbf{2}, \quad \omega_{-1}: \mathfrak{L}(\mathbb{R}) \rightarrow \mathfrak{L}(\mathbb{R}) \quad \text { and } \quad+: \mathfrak{L}(\mathbb{R}) \rightarrow \mathfrak{L}(\mathbb{R}) \oplus \mathfrak{L}(\mathbb{R})
$$

by $\varepsilon, \gamma$ and $\mu$, respectively. We also need the following well known result. Its proof is a straightforward checking of the commutativity of the diagrams given by group laws.

Proposition 7.1. The frame $\mathfrak{L}(\mathbb{R})$ with frame homomorphisms $\varepsilon, \gamma, \mu$ is an abelian localic group.

The general procedure of the preceding section applies to the case of $\mathfrak{L}(\mathbb{R})$ and $\mathfrak{L}(\mathbb{T})$ and the equalizer of Proposition 5.10 as we now check. Recall that the equalizer $e: \mathfrak{L}(\mathbb{T}) \rightarrow \mathfrak{L}(\mathbb{R})$ is given by

$$
(p, q) \mapsto \bigvee_{n \in \mathbb{Z}}(p+n, q+n)
$$

for each $p, q \in \mathbb{Q}$ and that we may identify the frame of the unit circle $\mathfrak{L}(\mathbb{T})$ with the complete sublattice $e(\mathfrak{L}(\mathbb{T}))$ of $\mathfrak{L}(\mathbb{R})$.

Now, by the results in the previous section, in order to have a localic group structure $(\bar{\mu}, \bar{\gamma}, \bar{\varepsilon})$ in $\mathfrak{L}(\mathbb{T})$ induced by $(\mathfrak{L}(\mathbb{R}), \mu, \gamma, \varepsilon)$ and $e: \mathfrak{L}(\mathbb{T}) \rightarrow \mathfrak{L}(\mathbb{R})$, we just have to confirm that $e$ satisfies identities (7.3.1) and (7.3.2), that is, 
(1) $\left(f \oplus 1_{\mathfrak{L}(\mathbb{R})}\right) \cdot \mu \cdot e=\left(1_{\mathfrak{L}(\mathbb{R})} \oplus 1_{\mathfrak{L}(\mathbb{R})}\right) \cdot \mu \cdot e=\left(1_{\mathfrak{L}(\mathbb{R})} \oplus f\right) \cdot \mu \cdot e$, and

(2) $f \cdot \gamma \cdot e=\gamma \cdot e$.

(1) First notice that if $p \geq q$ then $(p, q)=0$ and therefore

$$
\begin{aligned}
\left(\left(f \oplus 1_{\mathfrak{L}(\mathbb{R})}\right) \cdot \mu \cdot e\right)(p, q) & =\left(\left(1_{\mathfrak{L}(\mathbb{R})} \oplus 1_{\mathfrak{L}(\mathbb{R})}\right) \cdot \mu \cdot e\right)(p, q) \\
& =\left(\left(1_{\mathfrak{L}(\mathbb{R})} \oplus f\right) \cdot \mu \cdot e\right)(p, q)=\mathbb{O} .
\end{aligned}
$$

If $q-p>1$ then $(p, q)=1$ and so

$$
\begin{aligned}
\left(\left(f \oplus 1_{\mathfrak{L}(\mathbb{R})}\right) \cdot \mu \cdot e\right)(p, q) & =\left(\left(1_{\mathfrak{L}(\mathbb{R})} \oplus 1_{\mathfrak{L}(\mathbb{R})}\right) \cdot \mu \cdot e\right)(p, q) \\
& =\left(\left(1_{\mathfrak{L}(\mathbb{R})} \oplus f\right) \cdot \mu \cdot e\right)(p, q)=1 \oplus 1 .
\end{aligned}
$$

Finally, if $0<q-p \leq 1$ then

$$
(\mu \cdot e)(p, q)=\bigvee_{r \in \mathbb{Q}}\left(\left(r, r+\frac{q-p}{2}\right) \oplus\left(\bigvee_{n \in \mathbb{Z}}\left(p+n-r, \frac{p+q}{2}+n-r\right)\right)\right) .
$$

and therefore

$$
\begin{aligned}
\left(\left(f \oplus 1_{\mathfrak{L}(\mathbb{R})}\right)\right. & \cdot \mu \cdot e)(p, q)=\bigvee_{r \in \mathbb{Q}}\left(f\left(r, r+\frac{q-p}{2}\right) \oplus\left(\bigvee_{n \in \mathbb{Z}}\left(p+n-r, \frac{p+q}{2}+n-r\right)\right)\right) \\
& =\bigvee_{r \in \mathbb{Q}}\left(\left(r+1, r+\frac{q-p}{2}+1\right) \oplus\left(\bigvee_{n \in \mathbb{Z}}\left(p+n-r, \frac{p+q}{2}+n-r\right)\right)\right) \\
& =\bigvee_{s \in \mathbb{Q}}\left(\left(s, s+\frac{q-p}{2}\right) \oplus\left(\bigvee_{n \in \mathbb{Z}}\left(p+n-s+1, \frac{p+q}{2}+n-s+1\right)\right)\right) \\
& =\bigvee_{s \in \mathbb{Q}}\left(\left(s, s+\frac{q-p}{2}\right) \oplus\left(\bigvee_{m \in \mathbb{Z}}\left(p+m-s, \frac{p+q}{2}+m-s\right)\right)\right) \\
& =\left(\left(1_{\mathfrak{L}(\mathbb{R})} \oplus 1_{\mathfrak{L}(\mathbb{R})}\right) \cdot \mu \cdot e\right)(p, q) .
\end{aligned}
$$

Hence $\left(f \oplus 1_{\mathfrak{L}(\mathbb{R})}\right) \cdot \mu \cdot e=\left(1_{\mathfrak{L}(\mathbb{R})} \oplus 1_{\mathfrak{L}(\mathbb{R})}\right) \cdot \mu \cdot e$. Analogously, one can check that $\left(1_{\mathfrak{L}(\mathbb{R})} \oplus f\right) \cdot \mu \cdot e=\left(1_{\mathfrak{L}(\mathbb{R})} \oplus 1_{\mathfrak{L}(\mathbb{R})}\right) \cdot \mu \cdot e$.

(2) We have, for each $p, q \in \mathbb{Q}$,

$$
\begin{aligned}
(f \cdot \gamma \cdot e)(p, q) & =\bigvee_{n \in \mathbb{Z}}(f \cdot \gamma)(p+n, q+n)=\bigvee_{n \in \mathbb{Z}} f(-q-n,-p-n) \\
& =\bigvee_{n \in \mathbb{Z}}(-q-n+1,-p-n+1)=\bigvee_{n \in \mathbb{Z}}(-q-n,-p-n) \\
& =\bigvee_{n \in \mathbb{Z}} \gamma(p+n, q+n)=(\gamma \cdot e)(p, q) .
\end{aligned}
$$


By applying Theorem 6.6 we conclude that $(\mathfrak{L}(\mathbb{T}), \bar{\mu}, \bar{\gamma}, \bar{\varepsilon})$ is a localic group. In particular,

$$
\begin{aligned}
((e & \oplus e) \cdot \bar{\mu})(p, q)=(\mu \cdot e)(p, q)=\bigvee_{n \in \mathbb{Z}} \mu(p+n, q+n) \\
& =\bigvee_{n \in \mathbb{Z}} \bigvee_{r \in \mathbb{Q}}\left(\left(r, r+\frac{q-p}{2}\right) \oplus\left(p+n-r, \frac{p+q}{2}+n-r\right)\right) \\
& =\bigvee_{n, m \in \mathbb{Z}} \bigvee_{s \in[0,1)}\left(\left(m+s, m+s+\frac{q-p}{2}\right) \oplus\left(p+n-m-s, \frac{p+q}{2}+n-m-s\right)\right) \\
& =\bigvee_{s \in[0,1)}\left(\bigvee_{m \in \mathbb{Z}}\left(m+s, m+s+\frac{q-p}{2}\right) \oplus \bigvee_{k \in \mathbb{Z}}\left(p+k-s, \frac{p+q}{2}+k-s\right)\right) \\
& =(e \oplus e)\left(\bigvee_{s \in[0,1)}\left(\left(s, s+\frac{q-p}{2}\right) \oplus\left(p-s, \frac{p+q}{2}-s\right)\right)\right),
\end{aligned}
$$

hence $\bar{\mu}(p, q)=\bigvee_{s \in[0,1)}\left(\left(s, s+\frac{q-p}{2}\right) \oplus\left(p-s, \frac{p+q}{2}-s\right)\right)$, and

$$
\begin{aligned}
(e \cdot \bar{\gamma})(p, q) & =(\gamma \cdot e)(p, q)=\bigvee_{n \in \mathbb{Z}} \gamma(p+n, q+n)=\bigvee_{n \in \mathbb{Z}}(-q-n,-p-n) \\
& =e(-q,-p),
\end{aligned}
$$

hence $\bar{\gamma}(p, q)=(-q,-p)$, for every $p, q \in \mathbb{Q}$. One also has that $\bar{\varepsilon}(p, q)=1$ iff

$$
\varepsilon(e(p, q))=\varepsilon\left(\bigvee_{n \in \mathbb{Z}}(p-n, q-n)\right)=1 .
$$

Equivalently, $\bar{\varepsilon}(p, q)=1$ iff $0 \in \bigcup_{n \in \mathbb{Z}}\langle p+n, q+n\rangle$.

In conclusion, we have proved the following about the localic group of reals $(\mathfrak{L}(\mathbb{R}), \mu, \gamma, \varepsilon)$, the frame of the unit circle $\mathfrak{L}(\mathbb{T})$ and the inclusion frame homomorphism $e: \mathfrak{L}(\mathbb{T}) \rightarrow \mathfrak{L}(\mathbb{R})$ given by $(p, q) \mapsto \bigvee_{n \in \mathbb{Z}}(p+n, q+n)$ :

Theorem 7.2. If $\bar{\mu}: \mathfrak{L}(\mathbb{T}) \rightarrow \mathfrak{L}(\mathbb{T}) \oplus \mathfrak{L}(\mathbb{T})$ is the map such that $(e \oplus e) \cdot \bar{\mu}=\mu \cdot e$, $\bar{\gamma}: \mathfrak{L}(\mathbb{T}) \rightarrow \mathfrak{L}(\mathbb{T})$ is the map such that $e \cdot \bar{\gamma}=\gamma \cdot e$, and $\bar{\varepsilon}$ is the composite $\varepsilon \cdot e: \mathfrak{L}(\mathbb{T}) \rightarrow \mathbf{2}$, then

$$
(\mathfrak{L}(\mathbb{T}), \bar{\mu}, \bar{\gamma}, \bar{\varepsilon})
$$

is an abelian localic group.

Remarks 7.3. (1) Recall from Corollary 5.12 that $\mathfrak{L}(\mathbb{T})$ is isomorphic to $\mathcal{O}(\mathbb{R} / \mathbb{Z})$. Consequently, the localic group structure of $\mathfrak{L}(\mathbb{T})$ also arises from the canonical group structure of $\mathbb{R} / \mathbb{Z}$.

(2) Obviously the equalizer map $e: \mathfrak{L}(\mathbb{T}) \rightarrow \mathfrak{L}(\mathbb{R})$ is an LG-homomorphism $(\mathfrak{L}(\mathbb{T}), \bar{\mu}, \bar{\gamma}, \bar{\varepsilon}) \rightarrow(\mathfrak{L}(\mathbb{R}), \mu, \gamma, \varepsilon)$.

(3) Consider the neighbourhood filter of the unit of $\mathfrak{L}(\mathbb{T})$,

$$
N=\{s \in \mathfrak{L}(\mathbb{T})\} \mid \bar{\varepsilon}(s)=1\},
$$

and denote $\bar{\gamma}(a)$ by $a^{-1}$ for every $a \in \mathfrak{L}(\mathbb{T})$. Similarly as for $\mathfrak{L}(\mathbb{R})$, it follows from the results in $[6]$ that we have a canonical uniformity on $\mathfrak{L}(\mathbb{T})$, the left uniformity, generated by covers

$$
C_{s}=\left\{a \in \mathfrak{L}(\mathbb{T}) \mid a^{-1} a \leq s\right\} \quad(s \in N) .
$$


Analogously, the covers

$$
D_{s}=\left\{a \in \mathfrak{L}(\mathbb{T}) \mid a a^{-1} \leq s\right\} \quad(s \in N)
$$

and

$$
T_{s}=\left\{a \in \mathfrak{L}(\mathbb{T}) \mid\left(a^{-1} a\right) \vee\left(a a^{-1}\right) \leq s\right\} \quad(s \in N)
$$

form bases of uniformities, called the right and the two-sided uniformities of $\mathfrak{L}(\mathbb{T})$, respectively. Since $\mathfrak{L}(\mathbb{T})$ is abelian, the three uniformities coincide. It also follows from $[6]$ that $\mathfrak{L}(\mathbb{T})$ is complete in this uniformity.

Acknowledgements. The authors express their gratitude to the referee for several valuable comments and suggestions.

\section{References}

[1] B. Banaschewski, Compactification of frames, Math. Nachr. 149 (1990) 105-116.

[2] B. Banaschewski, The real numbers in pointfree topology, Textos de Matemática, vol. 12, University of Coimbra, 1997.

[3] B. Banaschewski, J. Gutiérrez García and J. Picado, Extended real functions in pointfree topology, J. Pure Appl. Algebra 216 (2012) 905-922.

[4] B. Banaschewski and C. J. Mulvey, A constructive proof of the StoneWeierstrass theorem, J. Pure Appl. Algebra 116 (1997) 25-40.

[5] B. Banaschewski and C. J. Mulvey, The spectral theory of commutative $C^{*}$-algebras: the constructive spectrum, Quaest. Math. 23 (2000) 425-464.

[6] B. Banaschewski and J. J. C. Vermeulen, On the completeness of localic groups, Comment. Math. Univ. Carolin. 40 (1999) 293-307.

[7] C. H. Dowker and D.P. Strauss, Sums in the category of frames, Houston J. Math. 3 (1977) 17-32.

[8] J. Gutiérrez García, T. Kubiak and J. Picado, Lower and upper regularizations of frame semicontinuous real functions, Algebra Univers. 60 (2009) $169-184$.

[9] J. Gutiérrez García, J. Picado and A. Pultr, Notes on point-free real functions and sublocales, in: Categorical Methods in Algebra and Topology, Textos de Matemática 46, pp. 167-200, University of Coimbra, 2014.

[10] S. S. Hong, Convergence in frames, Kyungpook Math. J. 35 (1995) 85-91.

[11] J. M. E. Hyland, Function spaces in the category of locales, in: Continuous Lattices (Proc. Bremen 1979, ed. by B. Banaschewski and R.-E. Hoffmann), Lecture Notes in Math. 871, pp. 264-281, Springer, Berlin, 1981. 
[12] J.R. Isbell, I. Kříž, A. Pultr and J. Rosický, Remarks on localic groups, in: Categorical Algebra and its Applications (Proc. Int. Conf. Louvain-LaNeuve 1987, ed. by F. Borceux), Lecture Notes in Math. 1348, pp. 154-172, Springer, Berlin, 1988.

[13] P. T. Johnstone, Stone Spaces, Cambridge Studies in Advanced Mathematics, vol. 3, Cambridge University Press, Cambridge, 1982.

[14] P. T. Johnstone, The point of pointless topology, Bull. Amer. Math. Soc. (N.S.) 8 (1983) 41-53.

[15] P. T. Johnstone, Vietoris locales and localic semilattices, in: Continuous lattices and their applications (Proc. Bremen, 1982), Lecture Notes in Pure and Appl. Math. 101, pp. 155-180, Dekker, New York, 1985.

[16] A. Joyal, Nouveaux fondaments de l'analyse, Lectures Montréal 1973 and 1974 (unpublished).

[17] O.K. Klinke, A presentation of the assembly of a frame by generators and relations exhibits its bitopological structure, Algebra Universalis 71 (2014) $55-64$.

[18] J. Madden, Frames associated with an abelian $\ell$-group, Trans. Amer. Math. Soc. 331 (1992) 265-279.

[19] I. Mozo Carollo, J. Gutiérrez García and J. Picado, On the Dedekind completion of function rings, Forum Math. (2015), doi: 10.1515/forum-20120095 .

[20] J. Picado and A. Pultr, Frames and locales: Topology without points, Frontiers in Mathematics, vol. 28, Springer, Basel , 2012.

[21] J. Picado and A. Pultr, Entourages, covers and localic groups, Appl. Categ. Struct. 21 (2013) 49-66.

[22] S. J. Vickers, Topology Via Logic, Cambridge Tracts in Theoretical Computer Science, vol. 5, Cambridge University Press, Cambridge, 1989. 